\title{
Abnormal endocrine pancreas function at birth in cystic fibrosis ferrets
}

\author{
Alicia K. Olivier, ${ }^{1}$ Yaling Yi, ${ }^{2}$ Xingshen Sun, ${ }^{2}$ Hongshu Sui, ${ }^{2}$ Bo Liang, ${ }^{2}$ Shanming Hu, ${ }^{3}$ Weiliang Xie, ${ }^{2}$ \\ John T. Fisher, ${ }^{2}$ Nicholas W. Keiser, ${ }^{2}$ Diana Lei, ${ }^{2}$ Weihong Zhou, ${ }^{2}$ Ziying Yan, ${ }^{2}$ Guiying Li, ${ }^{4}$ \\ Turan I.A. Evans, ${ }^{2}$ David K. Meyerholz, ${ }^{1}$ Kai Wang, ${ }^{5}$ Zoe A. Stewart, ${ }^{4}$ \\ Andrew W. Norris, ${ }^{3,6}$ and John F. Engelhardt ${ }^{2,6}$
}

\begin{abstract}
${ }^{1}$ Department of Pathology, ${ }^{2}$ Department of Anatomy and Cell Biology, ${ }^{3}$ Department of Pediatrics, ${ }^{4}$ Department of Surgery, ${ }^{5}$ Department of Biostatistics, College of Public Health, and ${ }^{6}$ Fraternal Order of Eagles Diabetes Research Center, University of lowa, lowa City, Iowa, USA.
\end{abstract}

\begin{abstract}
Diabetes is a common comorbidity in cystic fibrosis (CF) that worsens prognosis. The lack of an animal model for CF-related diabetes (CFRD) has made it difficult to dissect how the onset of pancreatic pathology influences the emergence of CFRD. We evaluated the structure and function of the neonatal CF endocrine pancreas using a new CFTR-knockout ferret model. Although CF kits are born with only mild exocrine pancreas disease, progressive exocrine and endocrine pancreatic loss during the first months of life was associated with pancreatic inflammation, spontaneous hyperglycemia, and glucose intolerance. Interestingly, prior to major exocrine pancreas disease, CF kits demonstrated significant abnormalities in blood glucose and insulin regulation, including diminished first-phase and accentuated peak insulin secretion in response to glucose, elevated peak glucose levels following glucose challenge, and variably elevated insulin and C-peptide levels in the nonfasted state. Although there was no difference in lobular insulin and glucagon expression between genotypes at birth, significant alterations in the frequencies of small and large islets were observed. Newborn cultured CF islets demonstrated dysregulated glucose-dependent insulin secretion in comparison to controls, suggesting intrinsic abnormalities in CF islets. These findings demonstrate that early abnormalities exist in the regulation of insulin secretion by the CF endocrine pancreas.
\end{abstract}

\section{Introduction}

Cystic fibrosis (CF) is caused by defects in the CF transmembrane conductance regulator (CFTR) chloride channel. Cystic fibrosisrelated diabetes (CFRD) is a common complication of CF and affects $20 \%-25 \%$ of adolescents and $40 \%-50 \%$ of individuals over 30 years of age $(1,2)$. CFRD is associated with worsening clinical status, including reduced pulmonary function, increased frequency of pulmonary exacerbations, and a decline in nutritional status (3-7). Furthermore, CFRD leads to increased mortality compared with CF patients without diabetes $(4,8)$. Thus, early diagnosis and treatment are vital to improving clinical outcome of CFRD patients.

While the pathophysiology of CFRD is multifactorial, delayed insulin secretion appears to be a key hallmark of disease progression (9-12), and the health of CF patients is improved by insulin therapy prior to and following diagnosis of overt diabetes $(13,14)$. Partial insulin deficiency occurs in part due to islet loss associated with exocrine pancreas disease (15-18). However, CF pancreata at CFRD autopsy demonstrate that remaining islets contain roughly half the number of insulin-positive cells found in non-CF controls $(17,18)$, and this degree of $\beta$ cell loss is thought to be insufficient to explain diabetes (19). Thus, insulin deficiency in CFRD is relative and not absolute. All stages of CFRD are characterized by abnormalities in circulating insulin levels $(10,12,20,21)$. Impaired first-phase insulin (IFPI) responses are common in CFRD patients, but also occur in approximately $50 \%$ of CF children with normal glucose tolerance (9). Current guidelines for CFRD screening

Authorship note: Alicia K. Olivier, Yaling Yi, and Xingshen Sun contributed equally to this work.

Conflict of interest: The authors have declared that no conflict of interest exists. Citation for this article: J Clin Invest. 2012;122(10):3755-3768. doi:10.1172/JCI60610. emphasize 2-hour blood glucose measurements following oral glucose tolerance testing (22).

Multiple non-pancreatic organ abnormalities, including those in the lung, liver, and intestine, may modify glucose disturbances in CF patients. For example, hepatic insulin resistance $(15,23,24)$ and/or enhanced peripheral insulin sensitivity have been observed in CF patients $(15,25,26)$. However, normal insulin sensitivity has also been reported in CF patients with impaired glucose tolerance or overt diabetes $(12,27-29)$. Altered entero-insular axis hormones such as cholecystokinin (CCK), glucagon-like peptide-1 (GLP-1), and glucose-dependent insulinotropic peptide (GIP) have been observed in CF patients (30-32) and may also play a role in CFRD, since each of these hormones participate in insulin regulation (33). Furthermore, chronic lung disease in CF is thought to contribute to abnormal glucose regulation and insulin resistance in concert with increasing metabolic demand (34).

Characterizing early structural and functional endocrine pancreas abnormalities that precede glucose intolerance in CF has been difficult due to the lack of an animal model in which CFRD spontaneously develops. Furthermore, to our knowledge, no clinical studies have evaluated CF glucose abnormalities in the first years of life. Cftr-knockout mice demonstrate enhanced sensitivity to low-dose streptozotocin-induced hyperglycemia, but are euglycemic in the absence of streptozotocin $(35,36)$. The recent development of CFTR-knockout models in the pig and ferret provides new avenues for investigating the pathogenesis of CFRD. Both CF pigs and ferrets develop intestinal, hepatic, pancreatic, and lung disease that is pathologically similar to various stages of disease in CF patients $(37,38)$; however, the progression of pancreatic disease in the two models is very different. CF pigs are born with significant exocrine pancreatic destruction, inflammation, and fibrosis 
A

A
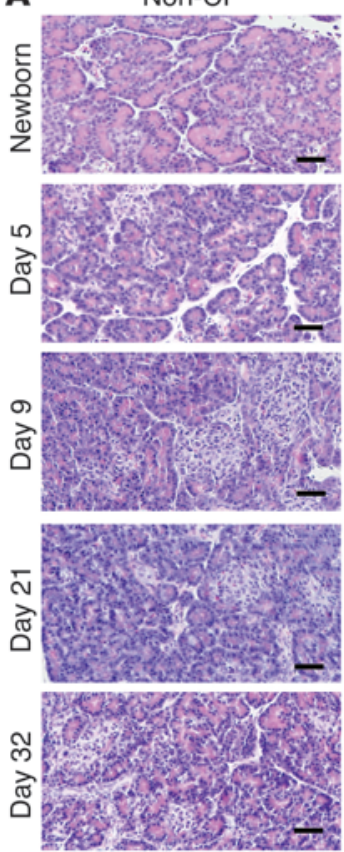

B

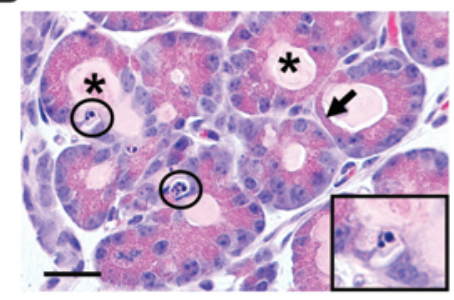

C

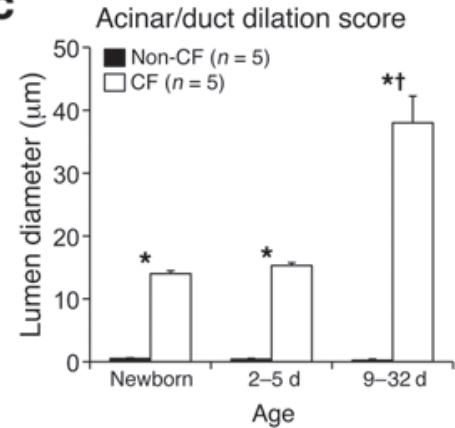

\section{Figure 1}

Progressive exocrine histopathology in the neonatal CF ferret pancreas. (A) Representative pancreatic histology from neonatal non-CF and CF ferrets. Multifocally the lumens of acini and lesser ducts of CF ferrets were dilated by pale eosinophilic material (*). Older CF animals exhibited loss of exocrine acini and replacement by fibrosis (arrows). Scale bars: $50 \mu \mathrm{m}$. (B) High magnification of acinar luminal dilation in a newborn CF pancreas exhibiting intraluminal material (*), loss of epithelial cytoplasmic zymogen granules (arrows), and apoptotic bodies (circled areas and higher-magnification inset) (scale bar: $50 \mu \mathrm{m}$; inset magnification: $\times 600$ ). (C) Acinar/duct dilation scores for specific age groups. Values are mean \pm SEM for $n$ independent animals. ${ }^{*} P<0.001$, age-matched CF versus non-CF controls for the various groupings; ${ }^{\dagger} P<0.001,9-32$ day versus newborn or $2-5$ day CF group; Kruskal-Wallis nonparametric 1-way ANOVA with a Dunn's post-test. characteristic of late-phase damage in CF patients, whereas CF ferrets have mild pancreatic histopathology at birth (38). For these reasons, the CF ferret model (39) may provide unique insights into the early pathogenesis of CFRD. CF newborn ferrets (kits) must be reared on antibiotics to prevent lung infections and on a proton pump blocker to raise gastrointestinal $\mathrm{pH}$ necessary for weight gain (40). As CF ferrets age, they require pancreatic enzyme supplementation to retain growth, similar to CF infants. CF kits are born with elevated markers of liver pathology, as occurs in human CF infants (41), and oral ursodeoxycholic acid (UDCA) therapy normalizes these markers in both species $(40,42,43)$. Thus, the CF ferret model has several characteristics similar to the human CF phenotype. While rearing of adult CF ferrets is challenging (40), the model also represents an opportunity to evaluate the early $\mathrm{CF}$ phenotype less easily approachable in human infants.

In the current study, we sought to determine whether alterations in insulin secretion and glucose homeostasis are present in newborn CF ferrets prior to structural loss of the endocrine and exocrine pancreas. Our studies demonstrate that fasted newborn CF ferrets have reduced first-phase insulin secretion and abnormal glucose tolerance. Structurally, newborn CF and non-CF endocrine pancreata were indistinguishable in terms of size and percent insulin and glucagon-expressing cells in islets. However, there was a difference in the distribution of large and small islets between genotypes and enhanced apoptosis in acinar and duct cells of CF animals at birth. Random blood insulin and C-peptide levels in the nonfasted state were widely variant and higher in newborn CF animals, whereas they were tightly regulated and lower in non-CF controls, suggesting that $\beta$ cell secretion of insulin is poorly regulated. Studies with isolated cultured islets from newborn animals confirmed dysregulated insulin secretion by CF $\beta$ cells, demonstrating elevated insulin secretion at low glucose and impaired induced insulin secretion to high glucose challenge, as compared with non-CF controls. In juvenile ferrets, progressive destruction of the exocrine and endocrine pancreas was associated with spontaneous hyperglycemia, glycosuria, and glucose intolerance. These findings in the CF ferret model demonstrate that alteration of endocrine pancreas function occurs very early in CF and is characterized, at least in part, by abnormal glucose responsiveness of the islet to secret insulin. Such alterations in the regulation of insulin secretion may be involved in the pathogenesis of CFRD.

\section{Results}

Progressive pancreatic inflammation and exocrine pancreas destruction are associated with impaired glycemic stability in CF ferrets. We previously reported that CF newborn ferrets have mild pancreatic pathology characterized by multifocal dilation of exocrine acini and lesser ducts by eosinophilic zymogen material (40). In the present study, we sought to better characterize age-dependent morphological changes in the pancreas in animals ranging from newborn to 1 month of age. Lumen dilation of acini and ducts resulted in epithelial changes including compression, attenuation, loss of zymogen granules, and nuclear morphologic changes consistent with apoptosis (Figure 1, $\mathrm{A}$ and $\mathrm{B}$ ). Immunohistochemical staining for cleaved caspase- 3 confirmed enhanced apoptosis in CF exocrine ducts and acini at birth (Supplemental Figure 1, A and B), suggesting that apoptotic damage in these regions occurs very early after birth coincident with duct dilatation. However, there was no significant difference in apoptosis of CF and non-CF newborn islets as measured by cleaved caspase-3/ insulin co-staining (Supplemental Figure 1, C and D).

By 21 days, dilated lumens were lined by cuboidal epithelial cells lacking zymogen granules, making discernment of acini versus ducts difficult, and were therefore termed acinoductular units. The lumen diameter of the acinoductular units was significantly larger in CF compared with non-CF pancreata in all age groups, with a marked increase between P9 and P32 compared with pancreata from younger CF kits (Figure 1C). By 21 days, there was loss of lobular parenchyma and replacement by bands of fibrous tissue 
A
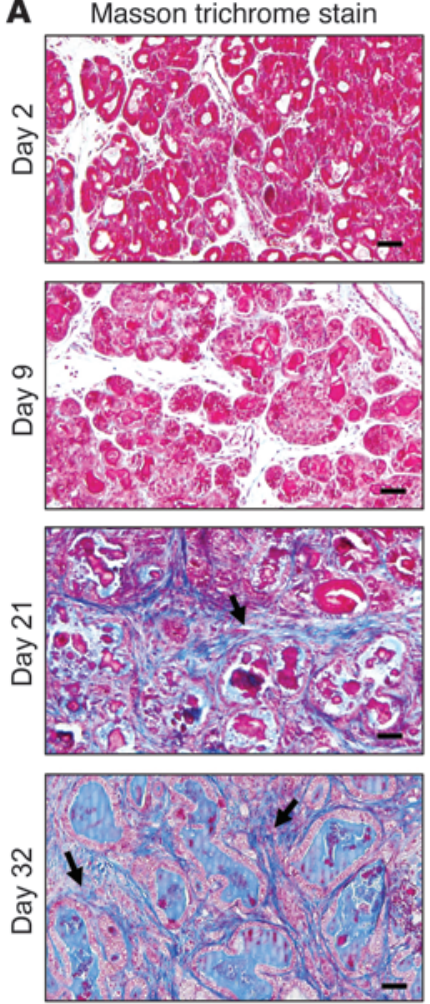

B
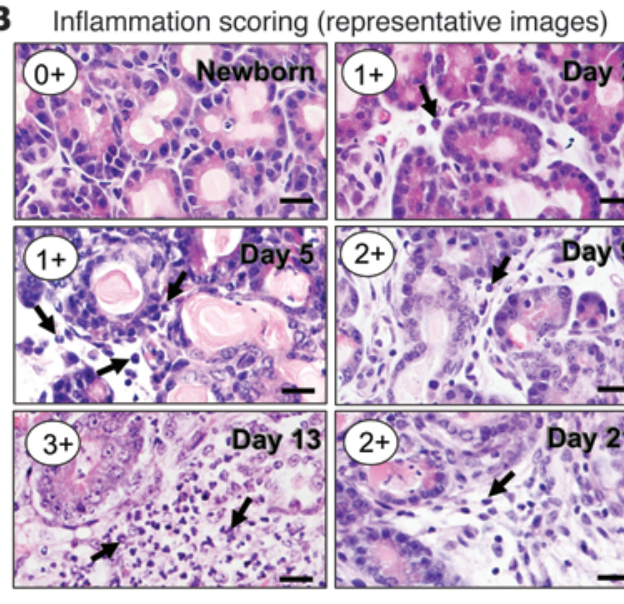

\section{C}

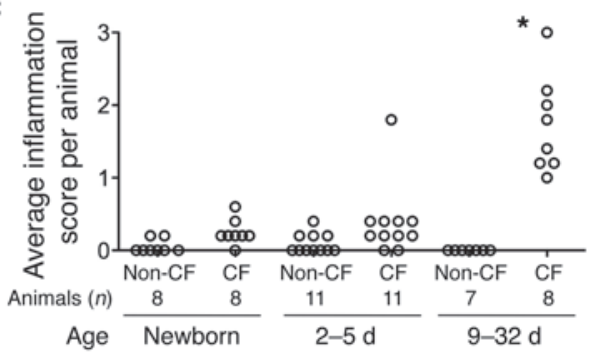

\section{Figure 2}

Progressive pancreatic fibrosis and inflammation in the neonatal CF ferret pancreas. (A) Time-dependent fibrosis of CF pancreata within the first month of life. Fibrous tissue stains blue by Masson trichrome stain in the interstitium (arrows). Note that the intraluminal material within dilated acinoductular units is nonspecific staining. Scale bars: $100 \mu \mathrm{m}$. (B and C) Inflammation scores were determined using a 0-3 scoring system based on number of interstitial inflammatory cells (arrows) per high-power field (HPF): 0 (no inflammatory cells/HPF), 1+ (1-9 inflammatory cells/HPF), 2+ (10-30 inflammatory cells/HPF), $3+$ (>30 inflammatory cells/ HPF). Degenerative neutrophils in acinoductular lumens, which increased with age, were not included in the inflammation scoring. (B) Histologic examples and scores for CF animals of various ages. Scale bars: $25 \mu \mathrm{m}$. (C) Average score for each animal evaluated for the various age groups, with $n$ total animals in each group. ${ }^{*} P<0.001$, 9- to 32-day-old CF versus non-CF controls, Kruskal-Wallis non-parametric 1-way ANOVA with a Dunn's post-test. Differences between genotypes in newborns or 2- to 5-dayold animals were not significant. that separated remnant dilated acinoductular units (Figure 2A). Pancreas sections were scored for inflammation on a scale of 0 to 3 (Figure 2B). No significant differences in inflammation were noted between genotypes in newborn or early neonatal pancreata (Figure 2C). However, inflammatory cell infiltration into the interstitium and peripancreatic adipose tissue was significantly higher in CF as compared with non-CF pancreata by 9-32 days and was characterized by the infiltration of neutrophils, macrophages, and lymphocytes (Figure 2C).

Major histopathologic changes in the exocrine pancreas preceded episodes of hyperglycemia and glycosuria, which emerged at 1-2 months of age (Figure 3 ). To control for the tailored (and thus varied) health care provided each CF animal, we evaluated glucose and glucose ratios among 3 sibling pairs of CF and nonCF animals matched for provided care and timing of blood collection. Roughly $44 \%$ of random nonfasted glucose measurements within the first 3 months of life were greater than $200 \mathrm{mg} / \mathrm{dl}$ in CF animals as compared with approximately $3 \%$ for sibling control littermates (Figure $3 \mathrm{~A}$ ). The $\mathrm{CF} /$ non-CF blood glucose ratio averaged $1.6 \pm 0.1$ across all time points and demonstrated a significant agedependent rise for the combined 3 sibling pairs $(P<0.0152)$ (Figure $3 \mathrm{~B}$ ). The onset of hyperglycemia (>200 mg/dl) ranged from 25 to 43 days and was preceded by a period of euglycemia (Figure 3C). As expected, hyperglycemia was associated with glycosuria in CF animals (ranging from 100 to $1,500 \mathrm{mg} / \mathrm{dl}$ ), and glucose was never observed in the urine of non-CF controls. These findings demonstrate that the progressive stages of histologic pancreatic damage in the CF ferret model correlates with impaired glycemic regulation and suggest this model may be useful for studying CFRD.

Glucose regulation by newborn and juvenile CF ferret. Exocrine pancreas insufficiency is a CFRD risk factor $(15,44)$. This observation underlies the prevailing hypothesis that CFRD arises from reduced islet mass and/or $\beta$ cell injury caused by nonspecific "bystander damage" to islets during exocrine pancreatic destruction and inflammation (17-19). Alternatively, a more direct role for CFTR in the regulation of the endocrine pancreas has been proposed $(35,36)$. Our above results demonstrating a correlation between hyperglycemia onset and histopathologic damage to the CF pancreas supports the bystander damage hypothesis (Figures 1-3), but does not disprove potential involvement of CFTR in regulating the endocrine pancreas. To explore this possibility, we characterized the early endocrine pancreas in newborn CF ferrets prior to overt exocrine pancreas pathology.

Fasted glucose tolerance tests (GTTs) on newborn animals 6-12 hours after birth demonstrated impaired glucose homeostasis in CF kits consisting of significantly elevated blood glucose at 30-120 minutes (Figure 4A) and significantly elevated glucose area under curve $\left(\mathrm{AUC}_{0-120 \mathrm{~min}}\right)$ (see Figure $4 \mathrm{~A}$ legend). CF kits at 5-11 days of age also demonstrated significantly altered glucose tolerance as compared with controls (Figure 4B), including significantly elevated $\mathrm{GTT}_{\text {Gluc5-30min }}$ glucose and $\mathrm{AUC}_{0-60 \mathrm{~min}}$ values in CF animals. Peak GTT values at 30 minutes were similar within a given genotype for newborn (CF $325 \pm 23 \mathrm{mg} / \mathrm{dl}$ vs. non-CF $218 \pm 20 \mathrm{mg} / \mathrm{dl}$ ) and 5- to 11 -day-old (CF $326 \pm 25 \mathrm{mg} / \mathrm{dl}$ vs. nonCF $215 \pm 23 \mathrm{mg} / \mathrm{dl}$ ) kits. This was also true for $\mathrm{AUC}_{\mathrm{Gluc} 0-120 \mathrm{~min}} \mathrm{val}-$ ues in both genotypes. Thus, there appears to be little change in glucose tolerance in CF kits during the first 2 weeks of life. These findings strongly support impaired glucose handling in neonatal CF kits prior to the emergence of overt pathology in the pancreas.

Progression of abnormalities in glucose handling with age was evaluated by mixed meal tolerance test (MMTT) in CF and nonCF animals ranging from 45 to 90 days of age. Results from these 

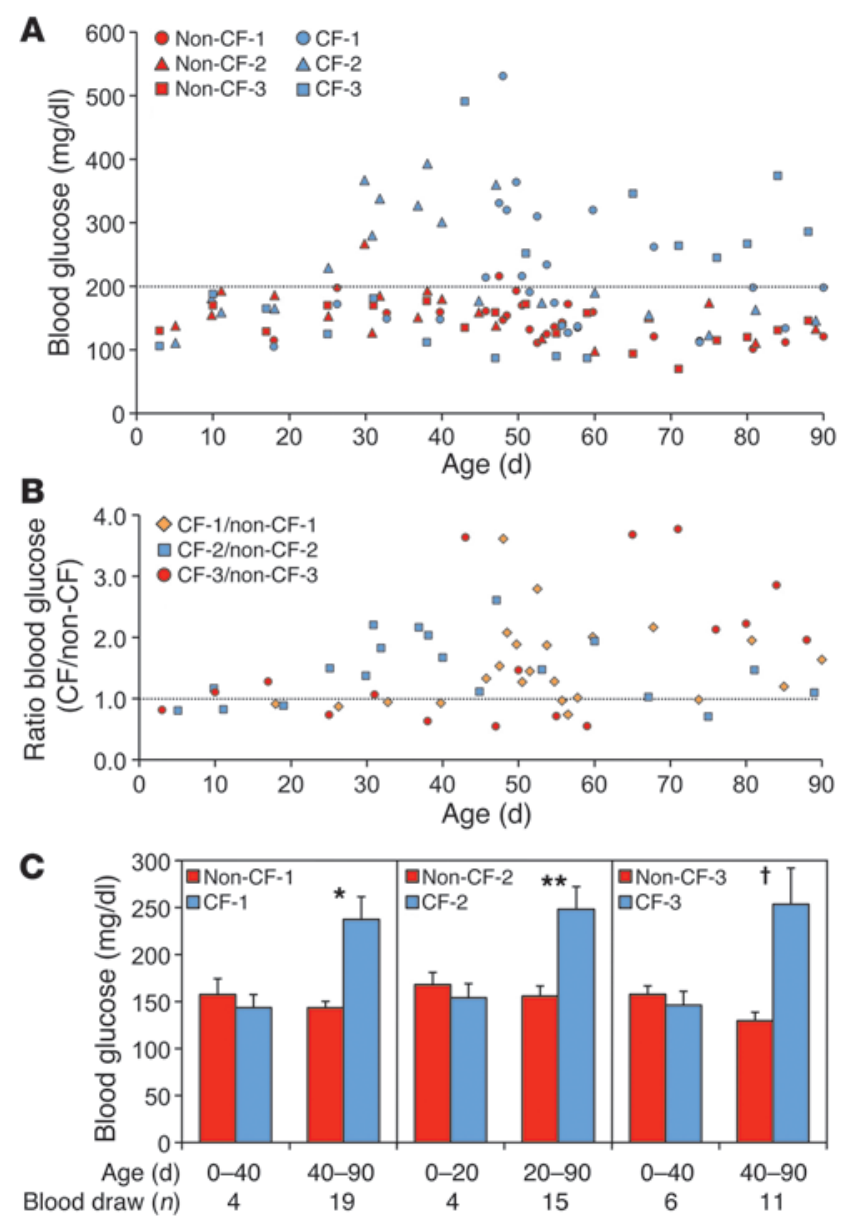

studies demonstrated a variety of abnormal glucose tolerance phenotypes in CF animals (Figure 4C). Although all CF animals demonstrated postprandial peak glucoses greater than $200 \mathrm{mg} / \mathrm{dl}$, the kinetics of the rise and fall in blood glucose was variable between animals. For example, $2 \mathrm{CF}$ animals retained significantly elevated Gluc $_{120 \mathrm{~min}}$ values $(270 \pm 44 \mathrm{mg} / \mathrm{dl}$ and $287 \pm 106 \mathrm{mg} / \mathrm{dl})$ as compared with non-CF controls $(129 \pm 10 \mathrm{mg} / \mathrm{dl}$ and $116 \pm 3 \mathrm{mg} / \mathrm{dl})$, with a profile similar to that shown in Figure 4C (left panel). By contrast, the third CF animal maintained normal average Gluc $_{120 \mathrm{~min}}$ values $(128 \pm 36 \mathrm{mg} / \mathrm{dl})$ as compared with non-CF control $(130 \pm 5 \mathrm{mg} / \mathrm{dl})$, with a profile similar to that shown in Figure 4C (right panel). Despite these observed differences in MMTT profiles of CF animals, $\mathrm{AUC}_{0-120 \mathrm{~min}}$ values for each of the $3 \mathrm{CF}$ animals were significantly greater than in non-CF controls (see Figure 4 legend), and the average glucose values at 30,60 , and 90 minutes after feeding were most significantly greater $(P<0.0001)$ in the $\mathrm{CF}$ as compared with the control group (Figure 4D). These studies demonstrate a progression in glucose abnormalities in CF ferrets with age.

Newborn CF ferrets have IFPI secretion. To evaluate whether impaired glucose tolerance in newborn CF ferrets might be due to altered insulin secretion, we quantified circulating insulin following a glucose or L-arginine challenge. L-Arginine stimulation tests (ASTs) have classically been used to evaluate first-phase insulin secretion (45). In this context, L-arginine leads to depolarization of the $\beta$ cell membrane and the immediate release of docked insulin granules at the membrane. L-Arginine challenge of newborn

\section{Figure 3}

CF ferrets develop impaired glycemic regulation at 1-2 months of life. (A) Blood glucose was measured on nonfasted, randomly fed CF and non-CF sibling pairs during the first 3 months of life. Each sibling pair was reared by the same jill, with blood draws taken simultaneously from each animal in the pair. Three independent $C F$ and non-CF sibling pairs are shown (Non-CF-1/CF-1, Non-CF-2/CF-2, and Non-CF-3/ CF-3). The percent of $n$ total readings above $200 \mathrm{mg} / \mathrm{dl}$ glucose for each animal was: Non-CF-1 $(4 \%, n=23)$, CF-1 $(44 \%, n=23)$, NonCF-2 $(5 \%, n=19)$, CF-2 $(42 \%, n=19)$, Non-CF-3 $(0 \%, n=17)$, CF-3 $(47 \%, n=17)$. Urine glucose in CF animals during episodes of hyperglycemia ranged from 100 to $1,500 \mathrm{mg} / \mathrm{dl}$ (CF-1: 8 of 9 urine samples tested positive; CF-2: 6 of 12 urine samples tested positive, CF-3: 1 of 12 urine samples tested positive). No glucose was found in any nonCF control urine measurements. (B) The ratio of CF to non-CF blood glucose for each sibling pair at each time point measured in A. Agedependent trends in $\mathrm{CF} /$ non-CF blood glucose ratios were evaluating using the log of the ratio of blood glucose and a mixed-effects model as described in Methods. There was a significant effect of age on the log of this ratio $(P<0.0152)$. (C) Average blood glucose levels for specific age windows prior to and following the first hyperglycemic episode (>200 mg/dl glucose) in the CF animal of each sibling pair. Values are mean \pm SEM. ${ }^{*} P<0.0007,{ }^{* *} P<0.0016,{ }^{\dagger} P<0.0137$, CF versus non-CF animals, nonparametric 2-tailed paired Wilcoxon test.
CF kits (3-hour-fasted) resulted in a significantly reduced 2-minute plasma insulin levels (5.7-fold) and insulin/glucose ratios (6.1-fold) in comparison to non-CF controls, despite similar blood glucose values (Figure 5, A-C). Similarly, reduced first-phase insulin response (6.5-fold) and insulin/glucose ratio (7.0-fold) were also observed in 3-hour-fasted newborn CF kits following an i.p. injection of glucose (Figure 5, D-F). By contrast, the level of plasma insulin at 30, 60, and 120 minutes following an i.p. injection of glucose to 3-hour-fasted kits was significantly greater in CF kits (6.8-, 14.8-, and 3.2-fold, respectively) as compared with non-CF kits (Figure 5G). Insulin/glucose ratios during the 30- and 60-minute measurements were also significantly elevated in CF kits as compared with non-CF kits (5.5- and 10.5-fold, respectively) (Figure $5 \mathrm{H}$ ); at these time points, $\mathrm{CF}$ kits were hyperglycemic relative to controls (Figure 4A). By contrast, at 120 minutes the insulin/ glucose ratio between genotypes was similar (Figure $5 \mathrm{H}$ ) despite persistent hyperglycemia in the CF group (Figure 4A), suggesting that elevated late-phase insulin secretion in CF animals is in response to hyperglycemia caused by an IFPI secretion.

Newborn CF ferrets have altered islet morphology but similar parenchymal insulin and glucagon expression. The observed insulin secretion abnormalities of newborn CF kits suggested that the endocrine pancreas was intact but functionally altered. To evaluate whether structural changes exist in the newborn CF ferret endocrine pancreas, we performed quantitative morphometry assessing insulin and glucagon. Interestingly, per insulin staining, newborn CF pancreata 
A

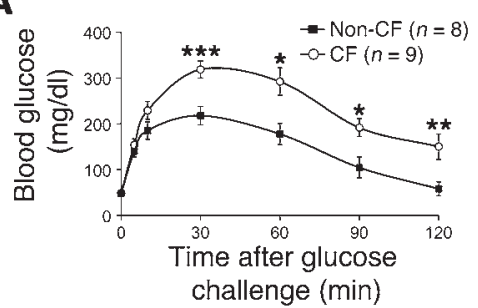

C
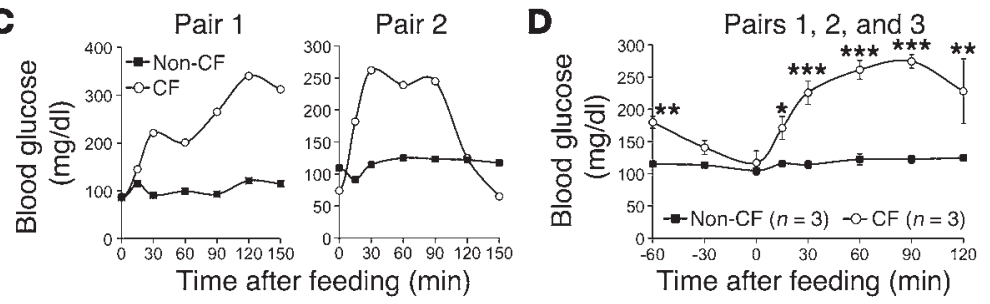

\section{Figure 4}

GTTs are abnormal in neonatal and juvenile CF ferrets. (A and B) GTTs were performed on 3-hour-fasted kits following i.p. injection of $2 \mathrm{~g} / \mathrm{kg} \mathrm{BW}$ glucose of (A) newborn and (B) 5- to 11-day-old CF and non-CF kits. (A) AUC ${ }_{0-120 m i n}$ was $C F$ $28,507 \pm 2221 \mathrm{mg} / \mathrm{dl} \cdot \mathrm{min}$ versus non-CF $17,940 \pm 2,011 \mathrm{mg} / \mathrm{dl} \cdot \min (P<0.0037)$. (B) AUC ${ }_{0-60 \mathrm{~min}}$ was CF 16,872 $\pm 1,518 \mathrm{mg} / \mathrm{dl} \cdot \min$ versus non-CF 10,688 \pm 956 $\mathrm{mg} / \mathrm{dl} \cdot \min (P<0.0087) ;$ AUC $_{0-120 \mathrm{~min}}$ was CF $29,160 \pm 3,903 \mathrm{mg} / \mathrm{dl} \cdot \mathrm{min}$ versus non-CF $18,113 \pm 1,629 \mathrm{mg} / \mathrm{dl} \cdot \mathrm{min}$ and not significantly different $(P<0.0823)$. Values in $\mathbf{A}$ and $\mathbf{B}$ represent mean \pm SEM for $n$ independent animals. (C and D) MMTTs were performed following a 3-hour fast on 3 groups of age-matched CF and non-CF siblings at 45-90 days of age. Each of 3 independent CF animals shown in Figure 3 was paired with 1-2 non-CF animals. (C) Individual MMTT assays demonstrating the diversity in blood glucose profiles from 2 independent pairs. (D) Average MMTT glucose values for the 3 paired CF/non-CF groups. Each paired group had 3-6 MMTT experiments performed, and the averages for CF and non-CF animals in each paired group were used to calculate the mean \pm SEM $(n=3)$. The average $\mathrm{AUC}_{0-120 \mathrm{~min}}$ for paired group 1 was CF 29,245 $\pm 5,920$ $\mathrm{mg} / \mathrm{dl} \cdot \mathrm{min}$ versus non-CF $14,973 \pm 1,105 \mathrm{mg} / \mathrm{dl} \cdot \min (P<0.0047)$; paired group 2, CF 27,454 $\pm 7652 \mathrm{mg} / \mathrm{dl} \cdot \min$ versus non-CF 13,108 $\pm 704 \mathrm{mg} / \mathrm{dl} \cdot \min$ $(P<0.0286)$; and paired group $3, \mathrm{CF} 27,325 \pm 3,792 \mathrm{mg} / \mathrm{dl} \cdot \mathrm{min}$ versus nonCF $14,813 \pm 729 \mathrm{mg} / \mathrm{dl} \cdot \min (P<0.0357)$. Differences between CF and non-CF animals at specific GTT time points (A and $\mathbf{B}$ ) and for all AUC analyses were evaluating using the nonparametric 2-tailed Mann-Whitney $U$ test ${ }^{* * *} P<0.001$, $\left.{ }^{* \star} P<0.005,{ }^{*} P<0.05\right)$. Two statistical analyses were performed on the log of blood glucose from individual MMTT measurements using a mixed-effects model as described in Methods. The first analysis assessed differences between CF and non-CF animals at specific MMTT time points in $\mathbf{D}\left({ }^{* *} P<0.0001,{ }^{* \star} P<0.01\right.$, $\left.{ }^{\star} P<0.05\right)$. The second analysis assessed differences in the overall trend in blood glucose following feeding of CF and non-CF animals $(P<0.0001, D)$.

had significantly more small islets and $\beta$ cell clusters ( 0.1 to less than $1.0 \mathrm{~mm}^{2}$ ) compared with non-CF pancreata (Figure 6, A-C). CF pancreata also had significantly fewer large islets $\left(>2 \mathrm{~mm}^{2}\right)$, but no difference in medium sized islets $\left(1-2 \mathrm{~mm}^{2}\right.$ ) (Figure 6, A, B, D, and $\mathrm{E}$ ). To determine whether the proliferation of insulin-positive cells in CF animals was altered in a manner that might impact islet size, we co-immunostained pancreatic tissue for proliferating cell nuclear antigen (PCNA, a proliferation marker) and insulin to determine the percentage of proliferating $\beta$ cells. The average percent proliferation for non-CF pancreatic $\beta$ cells was $22.6 \% \pm 1.3 \%$, and the average for $\mathrm{CF}$ pancreatic $\beta$ cells was $20.3 \% \pm 1.9 \%$, with no significant difference (Supplemental Figure 1, E and F). There were also no significant differences in PCNA staining observed for the various sizes of islets between genotypes (Supplemental Figure 1F).
To investigate other potential reasons for the variation in islet sizes between genotypes, we examined the expression of two transcription factors involved in islet neogenesis (PDX1 and SOX9). PDX1 and SOX9 are expressed in multipotent pancreatic cells but maintain selective expression in differentiated $\beta$ cells (PDX1), $\delta$ cells (PDX1), and duct cells (SOX9) (46). Although this is a highly debated topic, $\beta$ cells of the adult pancreas may originate from multiple sources, including self-replication of $\beta$ cells within islets, conversion of $\alpha$ cells to $\beta$ cells within islets, or transdifferentiation of PDX1-positive acinar and/or ducts cells (46). Analysis of PDX1 expression in insulin-positive cells demonstrated no differences in the distribution of these cells between various sizes of islets and genotype (Supplemental Figure 2, A and B). Furthermore, the frequency of PDX $1^{+}$insulin $^{-}$and $\mathrm{PDX} 1^{+}{ }^{+}$insulin ${ }^{+}$ cells within acinar/duct regions was also not different between genotypes (Supplemental Figure 2, C and D). As expected, SOX9 was highly expressed in cells of the ducts, with no discernible differences between genotypes, and expression was absent within islets of both genotypes (Supplemental Figure 2E). Thus, using these two markers, we could find no evidence for differences in expression patterns between genotypes that might suggest developmental abnormalities in the formation of islets.

We quantified the portion of the pancreas staining for insulin ( $\beta$ cells) and glucagon ( $\alpha$ cells), as their abundance might explain CF-associated alterations in glucose regulation. There was a small, but significant, reduction in insulin staining in samples from CF newborns when insulin staining was normalized to the total pancreatic area (Figure 7A), which by definition included acellular acinar and ductal lumenal contents. However, there was no significant difference when insulin staining was normalized to the total lobular area, which included only cellular areas of the pancreas (Figure 7B). The decrease in insulin staining when the data were normalized to total area was most likely a result of mild dilation of acini and ducts in the CF pancreas, which increased the relative pancreatic area. Supporting this hypothesis, the ratio of total lobular area to total pancreas area was significantly lower $(P<0.0004)$ for CF $(0.705 \pm 0.027, n=12)$ as compared with non-CF $(0.830 \pm 0.015, n=22)$ newborn samples. There was no significant difference in glucagon staining between newborn CF and non-CF pancreata using either total or lobular area normalization (Figure 7, C and D). Since histochemical staining indexes the spatial amount of cells as opposed to the quantity of the antigen, we also evaluated the total insulin content in newborn ferret pancreata. There was no significant difference in the insulin content, wet weight, or total protein content between CF and non-CF pancreata (Figure 7, E-G); however, CF pancreata demonstrated a trend toward higher insulin content.

To determine the effect of postnatal exocrine destruction on the loss of endocrine pancreas, we examined pancreata from older animals for insulin and glucagon staining. Similar to newborn CF pancreata, P2-P5 CF pancreata demonstrated a significant reduction in insulin staining normalized to total pancreas area, but not 

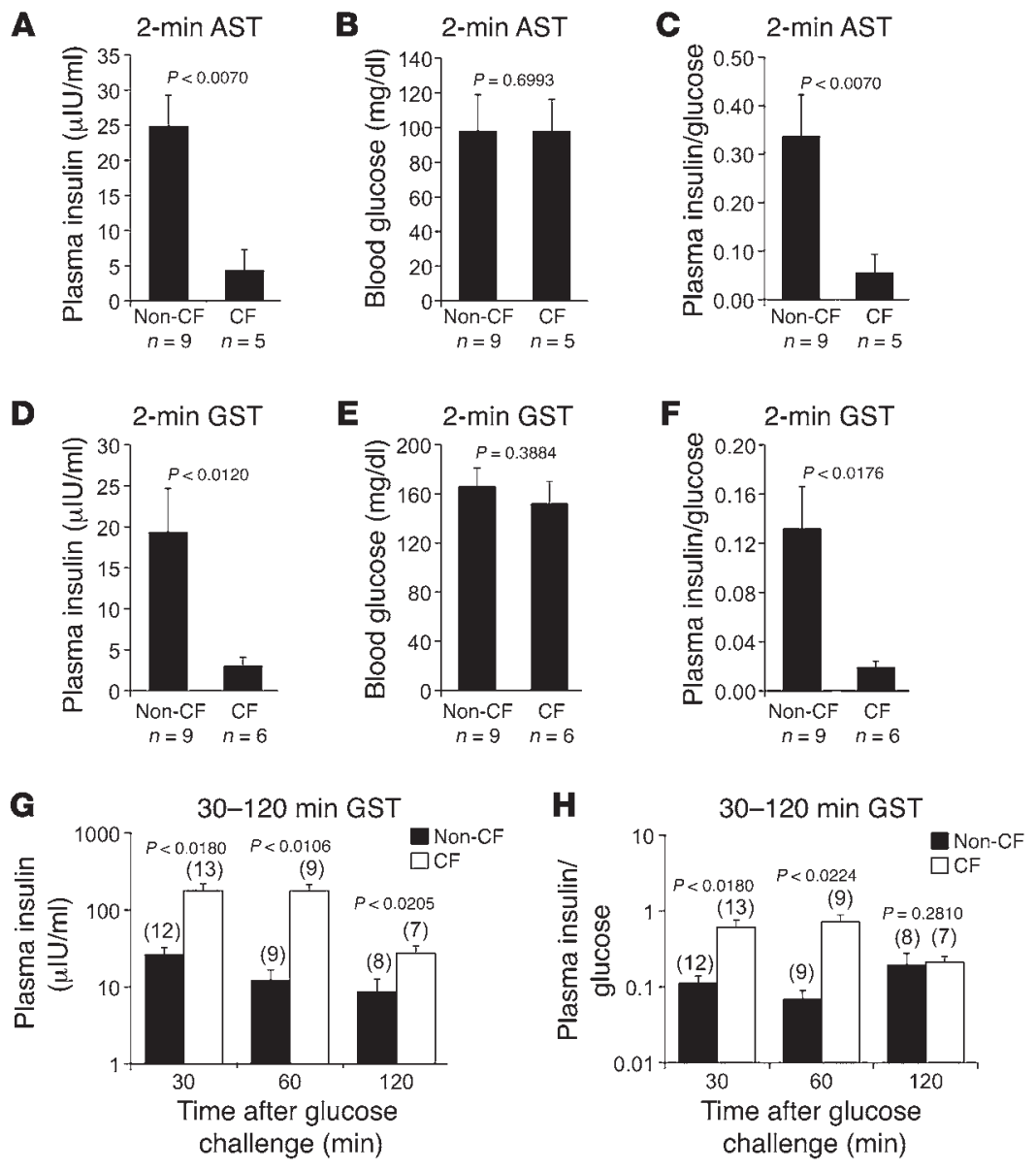

\begin{abstract}
Figure 5
First-phase insulin secretion is impaired in newborn fasted CF kits, while second-phase insulin secretion is accentuated. Glucose stimulation tests (GST) and L-arginine stimulation tests (AST) were performed on fasted newborn animals. (A-C) Blood insulin and glucose were measured in 3-hour-fasted newborn animals at 2 minutes following i.p. injection of L-arginine $(0.3 \mathrm{~g} / \mathrm{kg} \mathrm{BW})$. Values for insulin, glucose, and insulin/glucose ratios are shown. (D-F) Blood insulin and glucose were measured in 3-hour-fasted newborn animals at 2 minutes following i.p. injection of glucose (3 g/kg BW). Values for insulin, glucose, and insulin/glucose ratios are shown. ( $\mathbf{G}$ and $\mathbf{H})$ Blood insulin and glucose were measured in 3-hour-fasted newborn animals at 30,60 , and 120 minutes following i.p. injection of glucose (2 $\mathrm{g} / \mathrm{kg} \mathrm{BW})$. Values for (G) insulin and (H) insulin/glucose ratios are shown. Values are mean \pm SEM for $n$ independent animals. The number of animals tested $(n)$ in $\mathbf{G}$ and $\mathbf{H}$ is shown in parentheses above each bar. Differences were assessed using the nonparametric 2-tailed Mann-Whitney $U$ test.
\end{abstract}

when normalized to total lobular area of the pancreas (Figure 8, A and B). There was no significant change in glucagon staining in 2- to 5-day-old CF kits by either normalization method (Figure 8, $\mathrm{C}$ and $\mathrm{D})$, as was the case in newborn kits. By P9-P32, there was a significant reduction in both insulin and glucagon staining in $\mathrm{CF}$ pancreata compared with non-CF pancreata (Figure 8, E-G). Of note, in the older animals, it was not possible to normalize staining to lobular area given the loss of lobular tissue and ensuing fibrosis. This marked reduction in insulin and glucagon content in older CF animals preceded or coincided with the onset of glycemic instability in the nonfasted state (Figure 3).

Newborn CF ferrets have elevated and widely variant blood insulin and $C$-peptide in the nonfasted state. Because newborn CF ferrets demonstrated relative glucose intolerance, IFPI, and a heightened peak in later-phase insulin, it was important to evaluate how tightly kits could regulate their blood glucose and insulin levels during nonfasted conditions (i.e., intermittent spontaneous nursing). Interestingly, nonfasted CF newborn kits demonstrated significantly higher plasma insulin levels and insulin/glucose ratios as compared with non-CF controls, despite blood glucose levels that were indistinguishable between these two groups (Figure 9, A-C). Notably, CF plasma insulin levels and insulin/glucose ratios both demonstrated significantly increased variances by $F$ test $(P<0.0001)$, in comparison to non-CF controls. By contrast, there was no significant difference in the variance of blood glucose values between genotypes ( $F$ test, $P=0.3609$ ). The cause of the highly variable and elevated insulin levels in nonfasted CF newborns is of interest. Under nonfasted conditions, a relationship between glucose and insulin levels is expected. Indeed, in non-CF nonfasted newborns, glucose and insulin levels were positively correlated $(P<0.0468)$, such that lower glucose levels predicted lower insulin levels (Supplemental Figure 3A). By contrast, in CF nonfasted newborns, insulin did not vary with glucose $(P=0.7743)$, such that high insulin levels were observed even when glucose was low (Supplemental Figure 3A). These findings support the notion that insulin secretion by the newborn CF ferret pancreas is not properly regulated in a manner that extends beyond IFPI.

One potential reason for elevated and widely variant insulin levels in nonfasted CF kits could be increased glucocorticoids due to the physiological stress of $\mathrm{CF}$, thus promoting hepatic glucose production and insulin resistance. Since the major glucocorticoid in humans and ferrets is cortisol (47), we evaluated plasma cortisol levels in newborn randomly nursing CF and non-CF animals. Results from this analysis demonstrated no significant difference in plasma glucose, cortisol, or cortisol/glucose ratios between nonfasted CF and non-CF animals (Figure 9, D-F), demonstrating that changes in stress-induced glucocorticoid cortisol is likely not the cause of variant insulin levels in CF newborns.

While insulin is primarily cleared through the liver via an insulin receptor-mediated process (48), the proteolytic C-peptide fragment produced during insulin processing is primarily cleared through the kidney. C-peptide and insulin are secreted at a 1:1 molar ratio. Severe hepatic insulin resistance can be accompanied by reduced insulin receptor-mediated clearance and resultant hyperinsu- 
A

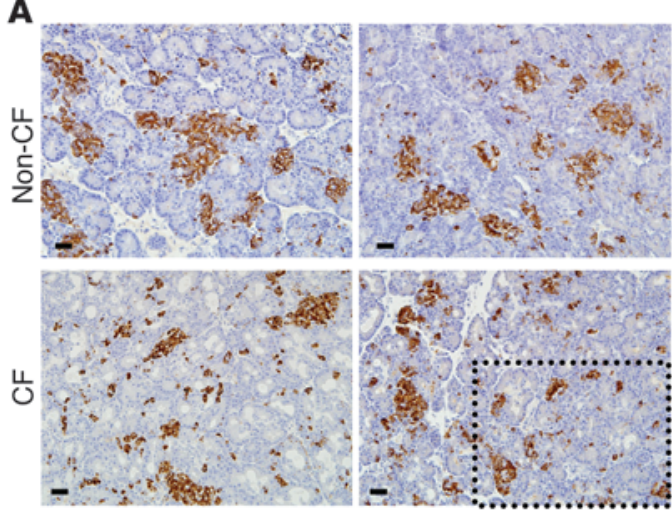

B

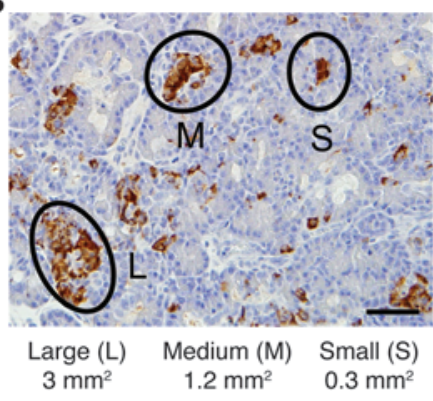

C

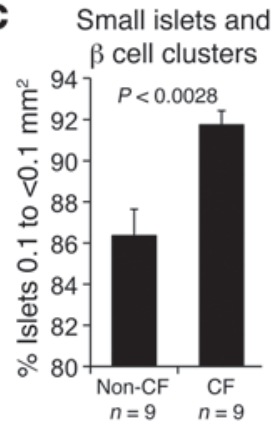

D

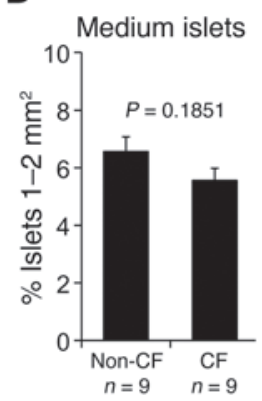

E

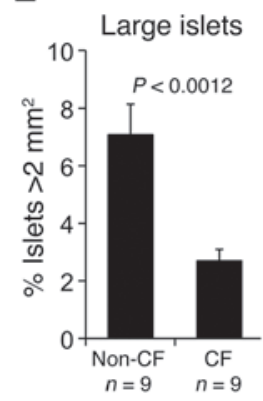

Figure 6

Differences in islet size between CF and nonCF newborn kits. (A) Representative images from insulin-stained pancreas sections from newborn non-CF and CF ferrets. Scale bar: $50 \mu \mathrm{m}$. (B) High magnification of boxed area in $\mathbf{A}$ demonstrating the size classification of small $\left(0.1\right.$ to $\left.<1 \mathrm{~mm}^{2}\right)$, medium $\left(1-2 \mathrm{~mm}^{2}\right)$, and large islets $\left(>3 \mathrm{~mm}^{2}\right)$. Scale bar: $50 \mu \mathrm{m}$. (C-E) The number of small (C), medium (D), and large islets (E) were quantified in non$\mathrm{CF}$ and CF pancreata, and the percentage for each group is shown. Nuclei are counterstained light blue. Values are mean \pm SEM for $n$ independent animals. Differences were assessed using the nonparametric 2-tailed Mann-Whitney $U$ test. linemia that exceeds C-peptide levels (49). Thus, in the absence of significant alterations of insulin clearance in CF animals, one would expect both plasma insulin and C-peptide levels to be similarly elevated in nursing $\mathrm{CF}$ as compared with non-CF kits. Indeed nonfasted CF newborn kits demonstrated significantly higher plasma C-peptide levels and C-peptide/glucose ratios as compared with non-CF controls (Figure 9, G and H), despite similar blood glucose levels in these two groups (Supplemental Figure 3B). These results indicate that the elevated insulin levels in CF kits represents bona fide insulin hypersecretion. Importantly, the molar ratio of plasma C-peptide/insulin was not significantly different between CF and non-CF animals (Figure 9I), although there was a trend toward lower molar ratios in the CF group. Like plasma insulin levels, C-peptide levels and C-peptide/glucose ratios both demonstrated significantly higher variances in CF animals $(P<0.0001)$. However, variances in plasma glucose or the molar ratio of $\mathrm{C}$-peptide/insulin were not significantly different between groups.

Isolated cultured CF islets improperly regulate insulin secretion. In vivo studies in newborn animals suggest that insulin secretion by the pancreas is improperly regulated. To evaluate whether islet-intrinsic CFTR defects influence insulin secretion, we utilized a previous method developed for neonatal pigs (50) to isolate and culture newborn islets from CF and non-CF ferrets. Insulin secretion over the course of 1 hour was then assayed under conditions of $2 \mathrm{mM}$ and $30 \mathrm{mM}$ glucose exposure. Similar to the trend toward higher levels of insulin found in the CF pancreas at birth (Figure 7E), isolated 9-day-cultured CF islets contained approximately 1.5 -fold more total insulin on average then non-CF islets (see Figure 9 legend for details). Interestingly, CF islets had a significantly higher (5.3-fold) percent insulin secretion at $2 \mathrm{mM}$ glucose in comparison to nonCF controls (Figure 9, J-L). Following stimulation with $30 \mathrm{mM}$ glucose, non-CF islets demonstrated a significant induction in percent insulin secretion (5.1-fold), while the percent insulin secretion for CF islets was not significant different at low $(4.1 \% \pm 0.9 \%)$ and high $(6.2 \% \pm 1.0 \%)$ glucose (Figure $9, \mathrm{~J}-\mathrm{L})$. These alterations were reflected in a 4-fold-reduced insulin secretory index (SI) in response to glucose challenge for CF islets as compared with non-CF islets (Figure 9, J and K). Similar to in vivo findings in nursing CF kits, the variance in values for percent insulin secretion by CF islets at low glucose was significantly greater than in their non-CF controls $(P<0.0001)$, suggesting dysregulated insulin secretion by the CF islet. Such findings provide strong support that islet-intrinsic CFTR defects alter glucose-responsive insulin secretion by the $\beta$ cell.

Insulin-mediated AKT activation is not altered in newborn CF ferret liver, skeletal muscle, or fat. Studies in cultured isolated islets suggest that a component of altered insulin secretion in CF may be intrinsic to the islet. However, it is also possible that insulin resistance could contribute to both the abnormal glucose tolerance and hyperinsulinemia observed in CF animals. The status of insulin resistance in CFRD is debated, with insulin sensitivity found to be reduced $(15,23,24)$, enhanced $(15,25,26)$, or normal $(12,27-29)$. Additionally, hepatic insulin resistance with enhanced peripheral insulin sensitivity is observed in a subset of CF patients that are exocrineinsufficient but lacking diabetes (15). To assess the state of insulin sensitivity in newborn CF and non-CF animals, we evaluated insulin-stimulated AKT phosphorylation in the liver, skeletal muscle, and adipose tissue as an indirect measure of insulin sensitivity, because hyperinsulinemic clamps are not feasible in newborn ferrets due to their size. Although altered AKT signaling has yet to be validated in the ferret as a marker of insulin resistance, reduction in insulin-stimulated AKT phosphorylation is a common feature of insulin resistance states in humans (51), pigs (52), and mice (53, 54). Results from these experiments demonstrated that newborn CF ferrets had levels of insulin-stimulated AKT phosphorylation 
A

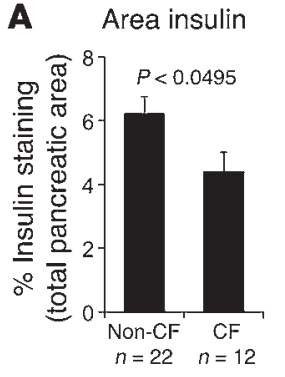

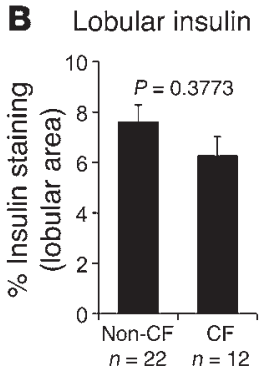

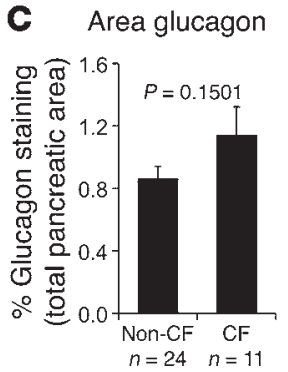

\section{Figure 7}

Insulin and glucagon content in newborn CF and non-CF pancreata. (A and B) Percent insulin staining in newborn CF and non-CF pancreas normalized to total pancreatic area $(\mathbf{A})$ and lobular area (B). (C and D) Percent glucagon staining in newborn CF and non-CF pancreas normalized to total pancreatic area (C) and lobular area (D). (E-G) Pancreas wet weight, total insulin content, and protein content of the newborn CF and non-CF pancreata. Values are the mean \pm SEM for $n$ independent animals. Differences were assessed using the nonparametric

2-tailed Mann-Whitney $U$ test.
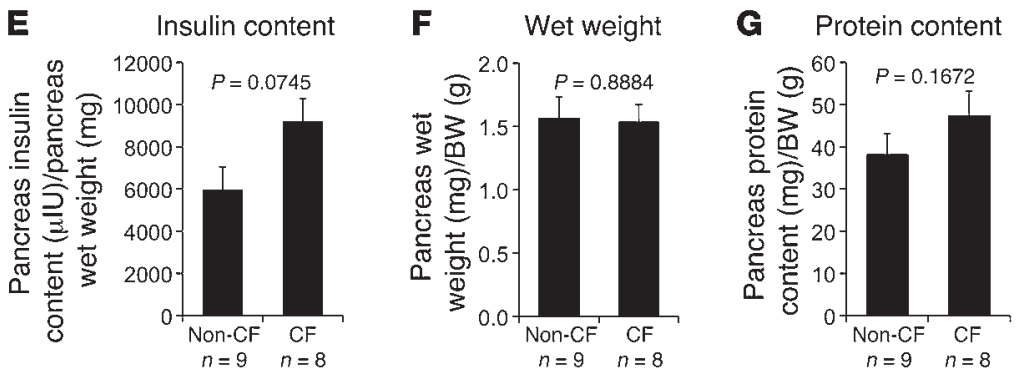

that were not significantly different from those in non-CF controls in all 3 tissues analyzed (Supplemental Figure 4). Although these findings do not rule out that potential CF-associated changes in hepatic or peripheral insulin sensitivity may impact insulin secretion or glucose tolerance, they do support the notion that impaired insulin clearance due to insulin resistance is not likely the cause of elevated insulin levels observed in fasted and fed CF animals.

CF liver disease predisposes patients to glucose abnormalities and diabetes $(55,56)$. However, it remains unclear whether neonatal liver pathologies in CF influence glucose and insulin regulation. Elevated levels in liver function tests (LFTs: alanine transaminase, aspartate transaminase, $\gamma$-glutamyltransferase, and bilirubin) are commonly observed in the majority of newborn CF infants (41, $42,57)$ and newborn CF ferrets (40). Neonatal elevations in LFTs observed in CF infants are generally thought to reflect cholestasis due to thickening of bile $(42,57,58)$. However, when liver biopsies were evaluated in CF neonates, there was no direct correlation between LFTs and histologic cholestasis (41). Whether this is a consequence of the focal nature of disease or an inherent component of the early liver CF pathobiology remains unclear (41). To further probe for potential hepatic pathologies in the CF ferret model that might impact early glucose and insulin abnormalities, we quantified bile duct plugging in canaliculi and bile ducts of $\mathrm{CF}$ and non-CF newborn animals. Results from these studies demonstrated significant increases in canalicular and bile duct plugging in CF animals as compared with controls (Supplemental Figure 5, A-D). Such findings correlate with elevations in plasma bilirubin observed in neonatal CF ferrets (40).

Fatty liver disease is not uncommon in human CF $(41,58)$, and liver dysfunction (including fatty liver) is often associated with insulin resistance and abnormal glucose metabolism in humans and mice $(49,59)$. Fatty liver (or steatosis) is characterized by excessive triglyceride deposition. Therefore, as a second index for potential alterations in liver metabolism, we assessed triglyceride content in the liver between CF and non-CF newborn ferrets. However, no significant difference in hepatic triglyceride content between genotypes was observed (Supplemental Figure 5E). Cumulatively, our finding on insulin/AKT signaling and hepatic triglyceride content suggest no significant differences between genotypes. By contrast, increased canalicular and bile duct plugging in CF animals suggests that early liver disease could in part contribute to alterations in insulin metabolism.

\section{Discussion}

The multiorgan nature of CF disease has made it difficult to dissect the early pathogenesis of CFRD. Glucose abnormalities and altered kinetics of insulin secretion are common in CF patients, occurring as early as in childhood. However, studies in infants and children younger than 6 years of age have yet to be performed. Our findings in the CFTR-knockout ferret demonstrate that insulin secretion is abnormally regulated very early during the course of CF disease and that islet-intrinsic CFTR defects cause dysregulated glucose-dependent insulin secretion. These abnormalities and other contributing factors, such as insulin resistance and pancreatic destruction, are likely important to the emergence of altered glucose metabolism in CF and the development of CFRD.

Most CF patients with altered and normal glucose tolerance demonstrate IFPI with delayed time to peak insulin (9-12). Such findings were similar in newborn CF ferrets. First-phase insulin secretion is critical for maintaining normal glycemia in response to glucose load, and IFPI is considered a key component of diabetes physiology (60). Our data suggest that IFPI is present very early in CF and may in part be facilitated by islet-intrinsic defects. The abnormalities in glucose tolerance that manifest at birth in fasted CF ferrets are most likely due to IFPI (Figure 4A and Figure $5, \mathrm{~A}-\mathrm{F})$. As expected with IFPI, delayed insulin responses following glucose stimulation of CF kits were accentuated (Figure 5G) at intermediate time points when hyperglycemia was observed during GTT. Whether the 10.5 -fold rise in insulin/glucose ratios at 60 minutes (Figure $5 \mathrm{H}$ ) at the peak of GTT insulin represents a pathologic overshoot is difficult to determine. However, the magnitude of overshoot was far greater for CF kits than that observed in CFRD adults (20) and children with abnormal glucose metabolism (61); insulin/glucose ratios at the time of delayed peak insulin in CF patients are similar or slightly lower than in control patients $(20,61,62)$. Thus, the magnitude of insulin overshoot observed in 

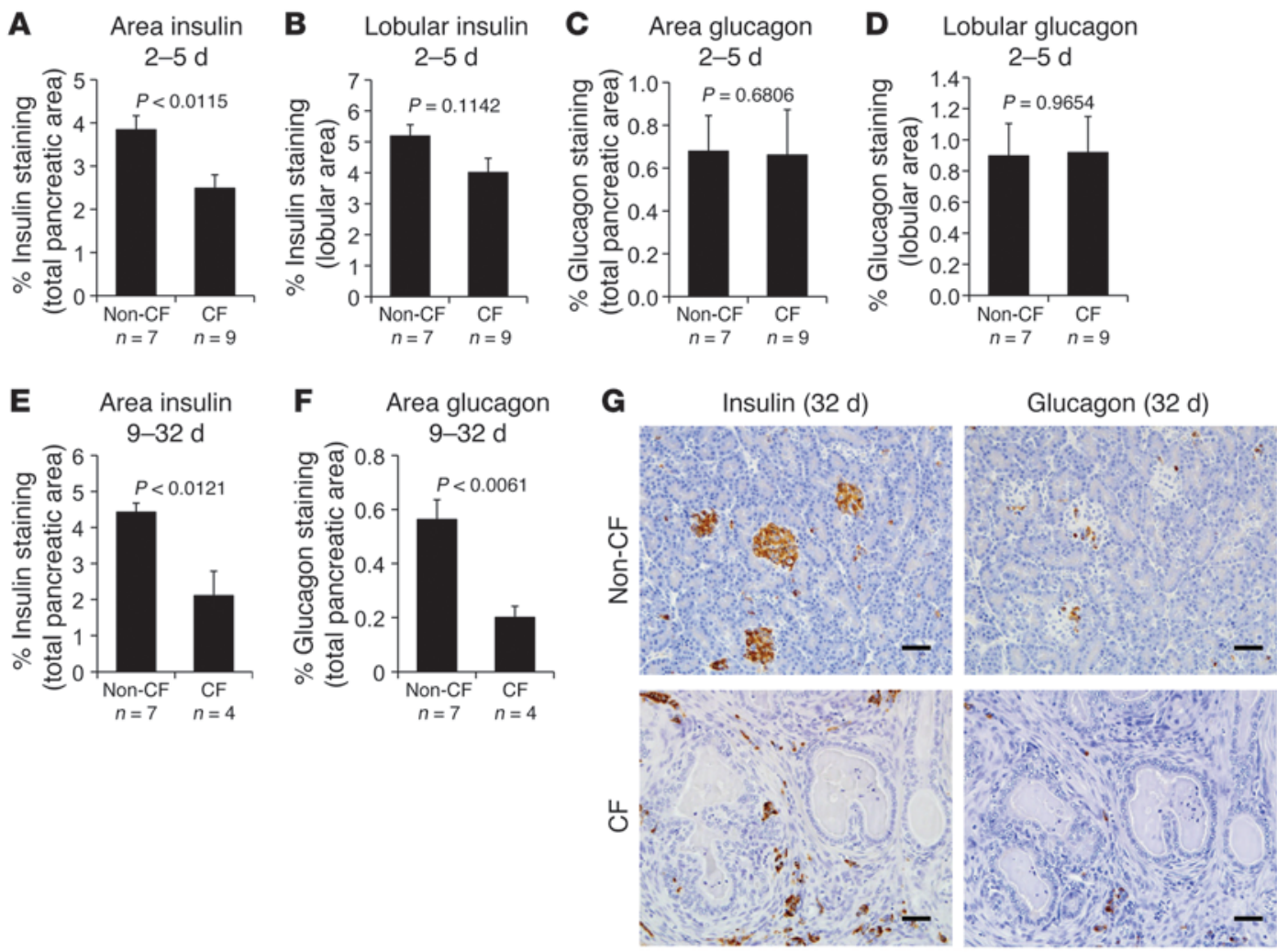

\section{Figure 8}

Insulin and glucagon content in neonatal CF and non-CF pancreata. (A and B) Percent insulin staining in pancreata from 2- to 5-day-old CF and non-CF kits normalized to total pancreatic area (A) and lobular area (B). (C and D) Percent glucagon staining in pancreata from 2- to 5-day-old CF and non-CF kits normalized to total pancreatic area (C) and lobular area (D). (E and F) Percent insulin (E) and glucagon (F) staining in pancreata from 9- to 32-day old CF and non-CF kits normalized to total area. (G) Representative images of 32-day-old CF and non-CF pancreata stained for insulin and glucagon. Scale bar: $50 \mu \mathrm{m}$. Values are mean \pm SEM for $n$ independent animals. Differences were assessed using the nonparametric 2-tailed Mann-Whitney $U$ test.

CF kits may be specific to the early neonatal period and physiologically appropriate, since hypoglycemia was not observed at 120 minutes, when insulin/glucose ratios had normalized to the level seen in non-CF animals (Figure 5H). One explanation for the high insulin levels would be systemic insulin resistance often observed in older CF patients $(15,23,24)$. However, we found no evidence to support this hypothesis through the evaluation of plasma cortisol levels (Figure 9, D-F) or insulin/AKT signaling in liver, muscle, and adipose tissue (Supplemental Figure 4). Nonetheless, the observed significantly enhanced bile plugging in hepatic canaliculi and bile ducts and non-significant trend toward reduced C-peptide/insulin molar ratios of CF animals suggest that hepatic abnormalities could impact insulin action and clearance in newborn CF ferrets.

The abnormal regulation of insulin in fasted glucose-challenged CF animals prompted us to evaluate how insulin secretion was regulated in randomly nursing kits. Given the IFPI observed in newborn CF kits, we hypothesized that glucose and insulin levels would be poorly regulated in fed animals. Indeed this was partly the case: plasma insulin, C-peptide, insulin/glucose, and C-peptide/glucose values were highly variable and on average much higher (8.5-, 6.5-, 14-, and 10-fold, respectively) in nonfasted CF kits compared with non-CF controls (Figure 9, A-C and G-I). Interestingly, $\mathrm{CF}$ animals appeared to partition into two populations in terms of plasma insulin and C-peptide levels, those with much higher levels and those with normal or near-normal levels. We speculate that this clustering may reflect differences in the postprandial timing of the blood sampling in this randomly nursing population. For example, those animals sampled shortly after feeding may mirror the hyper-insulin secretory state observed at 30-60 minutes during a GTT (Figure 5G). By contrast, animals that had not fed within 2-3 hours prior to sampling would be expected to have much lower insulin levels and normal insulin/ glucose ratios (Figure 5, G and $\mathrm{H}$ ). Because of the high insulin levels, we were interested in whether these randomly fed (nursed) CF kits would demonstrate a tendency toward lower blood glucose, which they did, but not to a statistically significant degree (Figure $9 \mathrm{~A}, P=0.0513)$. This suggests that the degree of hyperinsulinemia may tend toward being excessive. Consistent with this, insulin levels failed to decrease with lower glucose levels in nursing CF kits, while glucose and insulin levels were positively correlated in nursing non-CF kits (Supplemental Figure 3A). These tendencies observed in CF kits toward insulin oversecretion might play a role in the hypoglycemia that is observed in humans with $\mathrm{CF}(63,64)$.

Abnormalities intrinsic to the CF pancreas are another important factor that may influence insulin secretion. Although the newborn CF pancreas and CF cultured islets had approximately $50 \%$ more insulin content than non-CF controls, there was no significant difference in percent lobular insulin and glucagon staining 

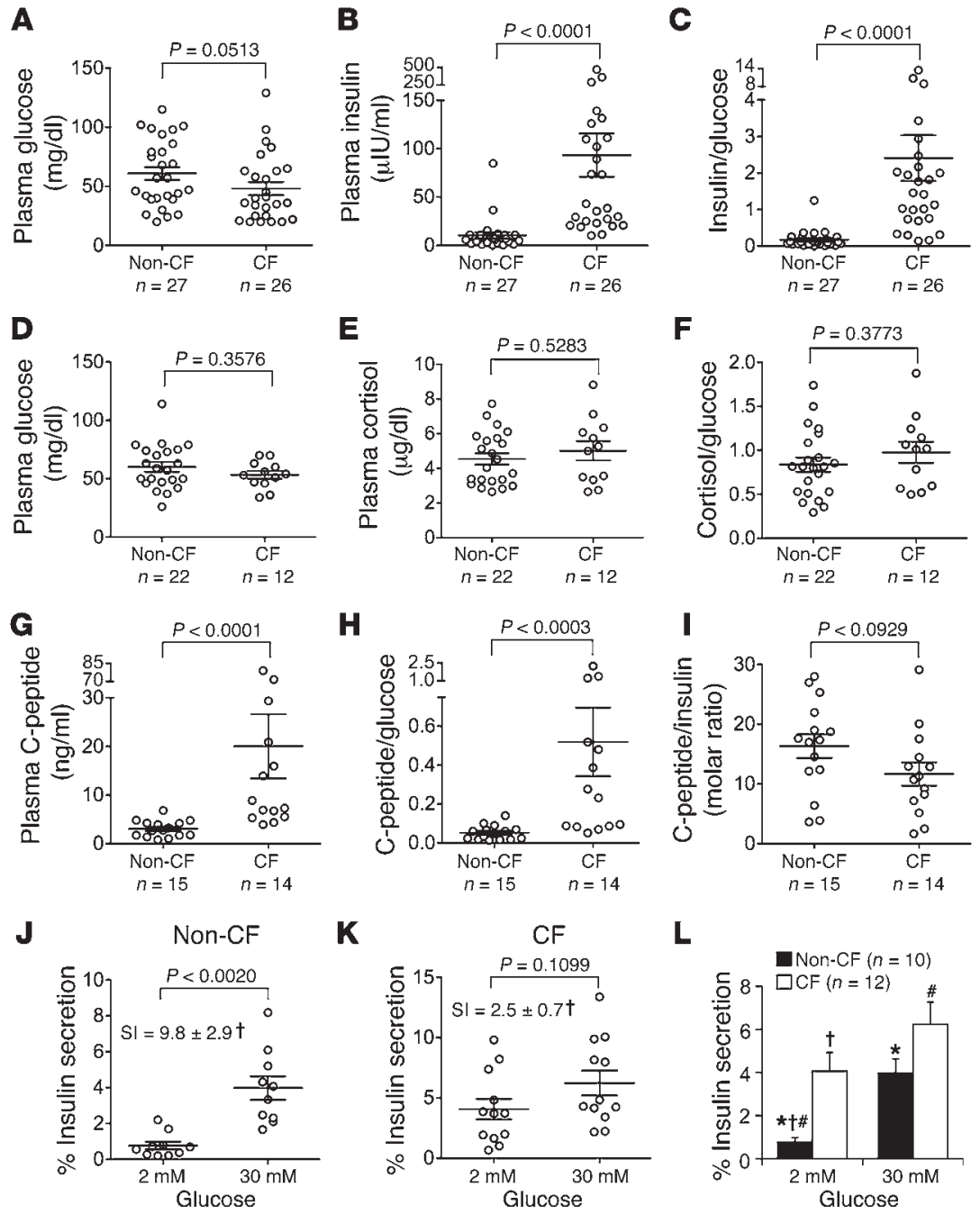

Figure 9

Abnormalities in islet insulin and C-peptide secretion occur in newborn CF ferrets. (A-I) Plasma collected from nursing newborn kits (6-12 hours after birth) was used to measure glucose, insulin, cortisol, and C-peptide. Since insufficient quantities of plasma could be obtained from 1 animal for all these measures, different cohorts were evaluated in A-C, D-F, and G-I. However, in a subset of animals in A-C there was sufficient plasma for C-peptide measurements, thus the cohort of animals in $\mathbf{G}-\mathbf{I}$ is included within A-C (for glucose, insulin, and insulin/glucose ratios for the cohort in $\mathbf{G}-\mathbf{I}$, see Supplemental Figure 3). ( $\mathbf{J}$ and $\mathbf{K}$ ) Insulin secretion by isolated cultured islets from (J) non-CF and (K) CF newborns. For each data point, pooled 9-day cultured islets were split into 2 groups of equally sized islets and incubated in the presence of 2 or $30 \mathrm{mM}$ glucose for 1 hour. The percent insulin secretion for each experiment and the average SI for all experiments were calculated as described in Methods. (L) Combined data from J and K. Supplemental Figure 6 shows representative insulin and glucagon immunofluorescence staining patterns for $\mathrm{CF}$ and non-CF islets. Values are mean \pm SEM for $n$ independent animals/experiments. Differences in A-I were assessed using a nonparametric 2-tailed Mann-Whitney $U$ test. Significantly higher variances in CF compared with non-CF values were observed for datasets in $\mathbf{B}, \mathbf{C}, \mathbf{G}$, and $\mathbf{H}(F$ test, $P<0.0001)$. No significant differences in variances were observed for $\mathbf{A}, \mathbf{D}-\mathbf{F}$, and $\mathbf{I}$. Differences in percent insulin secretion of $\mathbf{J}$ and $\mathbf{K}$ were assessed using a nonparametric 2-tailed paired Wilcoxon test, while differences in the secretory index $(\mathrm{SI})$ of $\mathrm{CF}$ and non-CF islets were assessed using the nonparametric 2-tailed Mann-Whitney test ( ${ }^{\dagger} P<0.0111$ comparing $S$ I values in $\mathbf{J}$ and $\mathbf{K}$. For $\mathbf{L}$, $\# P<0.001$, ${ }^{*} P<0.01,{ }^{\dagger} P<0.05$, Kruskal-Wallis nonparametric 1-way ANOVA with a Dunn's post-test. The average total insulin content of islets (secreted plus that remaining in the islet over the 1-hour period) was not significantly different between in CF $(1,198 \pm 302 \mu \mathrm{U}$ insulin, $n=12$ cultures) and non-CF (779 $\pm 184 \mu \mathrm{U}$ insulin, $n=10$ cultures) groups $(P=0.2485$, by 2-tailed Mann-Whitney $U$ test). between genotypes at birth (Figure 7). The observed differences in islet sizes between genotypes (Figure 6), however, indicate subtle changes in $\beta$ cell organization within CF pancreata that could impact insulin regulation. However, no differences in the distribution of two pancreas developmental markers (SOX9 and PDX1) were observed between genotypes, leaving the etiology of this finding still unknown. Whether this morphologic difference in islet size exists in CF human newborns is also unknown; however, previous reports have documented in utero and neonatal abnormalities in the development of the exocrine pancreas in $\mathrm{CF}$ individuals $(65,66)$. For example, exocrine acinar and duct lumens demonstrate a 10 -foldincreased volume in CF fetuses at 32-38 weeks after conception (65), a number that is strikingly similar to the 14-fold increase seen in newborn CF ferrets (Figure 1). Given the lower lobular/total area ratio in the CF ferret pancreas at birth and the similar pancreas wet weight/BW ratios of the genotypes, it is possible that CF pancreata have reduced total cellular mass at birth. Such a possibility in the CF pancreas could be due to loss of acinar cells and/or acinar cell compression. Additionally, the observed enhanced apoptosis in newborn CF ferret pancreatic acinar/duct cells demonstrates that early damage to the exocrine pancreas does occur, and this could potentially influence functions of the neonatal CF endocrine pancreas. These pathologies likely initiate prior to birth, based on CF human fetal studies, and could impact development of islets. While we observed no significant differences in $\beta$ cell apoptosis of CF and non-CF islets at birth, alternative mechanisms of $\beta$ cell injury not detected by activated caspase- 3 could indeed impact insulin secretion and glucose tolerance in CF neonates. Although we cannot rule out that small differences in $\beta$ cell apoptosis or replication at birth $(<1 \%-4 \%)$ might contribute to altered islet mass, the major contributing factor to reduction in islet mass in $\mathrm{CF}$ animals is likely the progressive pancreatic exocrine damage that occurs with age.

Although mild insulin resistance and early exocrine pancreatic damage may be isletextrinsic factors that contribute to altered insulin secretion in CF kits, studies in isolated cultured islets suggest that islet-intrinsic abnormalities alter glucose-responsiveness of the CF islet. As in nursing CF kits (Figure 9, A-C, and Supplemental Figure 3A), insulin secretion by CF islets is highly variable and less responsive to glucose stimulation in comparison to controls (Figure 9, J-L). Although IFPI 
in randomly nursing kits would be expected to lead to compensatory insulin secretion to maintain normal glycemia, abnormal glucose responsiveness of the $\mathrm{CF} \beta$ cell may also contribute to the inability of CF kits to properly regulate insulin secretion in the fed state. Interestingly, the intrinsic predisposition of isolated cultured CF islet to hypersecrete insulin at low glucose is not predicted by insulin levels in fasted CF kits, suggesting that the mechanistic basis of altered insulin secretion in CF kits is likely more complex than just IFPI. At low glucose, in vitro cultured CF islets secrete 5.2-fold more of their insulin content relative to non-CF controls, while also having impaired induction of insulin secretion to high glucose challenge. By contrast, the induction of insulin in fasted CF kits is accentuated at later time points, most likely due in large part to compensation for IFPI - CF newborn kits demonstrate a 38-fold rise in insulin/ glucose ratios between 2 and 60 minutes after glucose challenge as compared with a 1.9-fold decline in this ratio for non-CF animals for the same time frame. We hypothesize that an islet-extrinsic factor(s) may allow for normalization of insulin in fasted CF animals, suppressing the intrinsic islet tendency to over-secrete insulin at low glucose, and that such factor(s) are not present in the isolated $\mathrm{CF}$ islet. Excess abundance of such an insulin secretion-suppressive factor(s) in the fasted state could also account for the delayed insulin responses during transition to a fed state in CF animals.

Glucose abnormalities progressed with age in CF ferrets, demonstrating significant alterations in glucose excursion following MMTT. When these results are compared with CFRD clinical classifications using oral GTTs, older CF ferrets demonstrated two MMTT phenotypes, including impaired glucose tolerance and indeterminate glycemia (Figure 4C). Although MMTTs are not a pure measure of glucose tolerance, being influenced by enteroinsular axis hormones that could be altered in a CF-specific manner, the MMTT glucose excursion curves were similar to those observed in CFRD patients not only following oral GTTs but also following a meal $(20,61)$. Interestingly, progressive destruction and inflammation of the exocrine pancreas in older CF ferrets (Figure 2) were associated with the appearance of spontaneous hyperglycemia and glycosuria (Figure 3). These older CF ferrets demonstrated a $52 \%$ loss of insulin-positive cells (Figure $8 \mathrm{E}$ ), which is very similar to the approximately $50 \%$ loss in $\beta$ cells seen at autopsy in CF patients, including those with overt diabetes and glucose intolerance (18). Relatively little is know about how early phases of pancreatic inflammation and exocrine decline impact glycemic regulation in CF patients. In non-CF children, a single episode of acute pancreatitis can lead to insulin requiring diabetes mellitus (67). Pancreatic-sufficient CF patients are most susceptible to acute pancreatitis during adolescence and young adulthood (68-70), while pancreatic-insufficient patients rarely develop acute pancreatitis. Based on the CF ferret, inflammation association with the onset of exocrine pancreas decline appears closely linked to spontaneous glucose abnormalities and worsening of glucose tolerance.

In summary, our studies in CFTR-knockout ferrets suggest that abnormalities in insulin secretion and glucose metabolism may occur very early in CF patients. Although multiple factors likely contribute to altered insulin regulation in $\mathrm{CF}$, including exocrine pancreas inflammation and changes in systemic insulin resistance and sensitivity, our results suggest, for the first time to our knowledge, that islet-intrinsic abnormalities in CF influence glucose-mediated regulation of insulin. Future studies in the CF ferret model may help to clarify the mechanism of altered insulin secretion and may lead to improved therapies for human CFRD patients.

\section{Methods}

Ferret breeding and rearing. The previously described CFTR exon 10-disrupted ferret model was used for all studies (39). CFTR ${ }^{+/-}$hobs and jills were bred at Marshall Farms and shipped to the University of Iowa for birth at 28 days gestation ( 2 weeks prior to whelping at 42 days gestation). Litters of $\mathrm{CFTR}^{+/+}, \mathrm{CFTR}^{+/-}$, and $\mathrm{CFTR}^{-/-}$kits were rapidly genotyped at birth by PCR using modifications to a previously described method (40). A small piece of tail tissue (0.1-0.2 cm in length) from each kit was lysed by heating at $95^{\circ} \mathrm{C}$ in $50 \mu l$ lysis buffer $(25 \mathrm{mM} \mathrm{NaOH}, 0.2 \mathrm{mM}$ EDTA) for 40 minutes. The lysate was then neutralized by adding $50 \mu \mathrm{l}$ of $40 \mathrm{mM}$ Tris- $\mathrm{HCl} \mathrm{pH} 7.4$ and clarified by centrifuging at $10,000 \mathrm{~g}$ for 10 minutes. One microliter of supernatant was then used directly for the PCR using the genotyping reaction and primers previously described (40). When litters were reared, a protocol previously described was used with minor modification (40). This involved gavage feeding with 150-200 $\mu$ l Elecare (Abbott Laboratories) containing 10\% GoLYTELY, ursodeoxycholic acid (5 mg/kg), and omeprazole $(5 \mathrm{mg} / \mathrm{kg}) 4$ times daily. Kits also were free to nurse and, depending on weight gain, were supplemented with 2-4 additional Elecare $10 \%$ GoLYTELY gavages daily. All animals were reared from birth on the antibiotics metronidazole ( $5 \mathrm{mg} / \mathrm{kg}$, 4 times daily) and Zosyn $(4.0 \mathrm{mg} / \mathrm{kg}$, twice daily) with each injection given in $100 \mu \mathrm{l}$ saline s.c. As animals gained weight, the amount of saline was increased proportionally with BW for each injection. Because CF kits are highly susceptible to lung infection, an additional antibiotic was used if kits began to lose weight during a 2-day period. These two antibiotics included enrofloxacin $(1.5 \mathrm{mg} / \mathrm{kg}$, twice daily) and ceftazidime ( $10 \mathrm{mg} / \mathrm{kg}$, 4 times daily). Control non-CF siblings $\left(\mathrm{CFTR}^{+/-}\right.$or $\left.\mathrm{CFTR}^{+/+}\right)$were treated identically during the rearing process.

Histology and immunohistochemistry. At necropsy, pancreata were removed and fixed in $10 \%$ neutral buffered formalin. Tissues were routinely processed, embedded in paraffin, and sectioned at $4 \mu \mathrm{m}$. Sections were stained with hematoxylin and eosin for light microscopy. Formalin-fixed, paraffinembedded pancreata were stained by immunohistochemistry for insulin (MP Biomedicals), glucagon (MP Biomedicals), PCNA (Dako), PDX1 (Millipore), and SOX9 (Millipore). For single stains, tissue sections were deparaffinized in xylene and rehydrated through graded alcohols. Slides were treated with proteinase $\mathrm{K}$ for 5 minutes. Endogenous peroxidase activity was quenched by immersing slides in 3\% hydrogen peroxide for 8 minutes. After blocking in Background Buster (Innovex Biosciences) for 30 minutes, tissues were incubated for 1 hour at room temperature with the primary antibody: guinea pig anti-insulin (1:4,000, 60 minutes) or rabbit anti-glucagon (1:50, 60 minutes). Rabbit anti-guinea pig HRP (Dako) was applied to the insulin slides at 1:500 for 30 minutes. Rabbit EnVision (Dako) secondary antibody was applied for 30 minutes to all slides. Slides were developed with DAB Plus (Dako) for 5 minutes, followed by DAB Enhancer (Dako) for 3 minutes. Slides were then counterstained and coverslipped. Negative control slides were prepared by substituting primary antibody with buffer. Dual staining for insulin (1:4,000, 60 minutes)/PCNA (1:4,500, 15 minutes), insulin (1:4,000, 60 minutes)/PDX1 (1:4,000, 60 minutes), and insulin (1:4,000, 60 minutes)/SOX9 (1:4,000, 60 minutes) was performed using the EnVision G2 Doublestain System (Dako). Following deparaffinization, slides were retrieved in citrate buffer ( $\mathrm{pH}$ 6.0) in a Decloaker (Biocare) for 20 minutes. The dual staining kit directions were then followed.

Morphometric analysis. Whole-section images of insulin- and glucagonstained pancreas were obtained using a BX-51 microscope with CellSens digital imaging software (Olympus) at $\times 200$. Images were analyzed using ImageJ software (http://rsbweb.nih.gov/ij/). Insulin- and glucagon-immunoreactive cells had diffuse cytoplasmic staining. Images were converted to an RGB stack, and the staining was thresholded in the blue channel identically for all samples for each stain. The percent parenchymal staining of insulin and glucagon was determined by dividing the stained area by 
(a) the total pancreatic area, including acellular duct and acinar lumens, and (b) the total lobular area, including endocrine and exocrine parenchyma (which includes only the cellular area and excludes acellular duct and acinar lumens). For measurement of the lobular tissue area, the section was thresholded and area measured in the red channel. For islet morphometry analysis, the insulin-stained sections were thresholded in the blue channel, and particles were counted as defined by particle size restrictions. Islets were designated by area: small ( 0.1 to less than $1.0 \mathrm{~mm}^{2}$, medium $(1.0-2.0$ $\left.\mathrm{mm}^{2}\right)$, and large $\left(>2.0 \mathrm{~mm}^{2}\right)$. Islets sizes and insulin/glucagon staining were quantified from one section per animal taken from a similar plane of section and digitally scanned in its entirety. The entire section was quantified from each animal. On average, approximately 550 islets were quantified per section/animal. The percentage of the islet sizes was then determined based on the total number of islets counted in the section, and the percent insulin or glucagon staining (lobular and total area) was calculated as described above. For quantification of immunohistochemical staining patterns (PCNA/insulin, activated caspase-3/insulin, and PDX1/insulin), sections were imaged at $\times 400$, and 5 images per section ( 1 section per animal) were quantified. For each section, approximately 300 insulin-positive islet cells were counted, and islet size was documented. The insulin-positive islet cells that were also PCNA, activated caspase-3, or PDX1 positive were counted and converted to a percent of total cells. To quantify acinar/duct apoptosis, caspase-3-positive cells were counted in five $\times 400$ fields $(\sim 1,200$ total acinar/duct cells per section). For quantification of PDX1 staining in acinar/duct regions of the pancreas, PDX1-positive or PDX1/insulin dualpositive cells were counted for each section as a fraction of total nuclei $(\sim 500$ acinar/duct cells per section) and values converted to a percentage. For quantification of the acinar/duct dilation score, images were taken at $\times 200$ and all lumen diameters measured. Two images per section were quantified. Inflammation was scored on a scale of 0 to 3 based on the number of interstitial inflammatory cells per five $\times 400$ fields: 0 (no inflammatory cells), 1 (<10 inflammatory cells), 2 (10-30 inflammatory cells), and 3 ( $>30$ inflammatory cells). Degenerative neutrophils, occasionally found within dilated acinoductular lumens of younger animals and always seen in the acinoductular lumens of older animals, were not included in the inflammation scoring.

GTTs. GTTs were carried out on 3-hour-fasted newborn (6-12 hours after birth) and 5- to 11-day-old kits. CF $\left(\mathrm{CFTR}^{-/-}\right)$and non-CF $\left(\mathrm{CFTR}^{+/+}\right.$and $\left.\mathrm{CFTR}^{+/-}\right)$kits are designated as experimental and control groups, respectively. Kits were injected (i.p.) with glucose $(2 \mathrm{~g} / \mathrm{kg} \mathrm{BW})$, and blood glucose levels were measured from tail pin prick immediately before and 5, 10, 30, 60,90 , and 120 minutes after the glucose injection using an automatic blood glucose monitor (One Touch LifeScan). AUC was calculated using the trapezoid rule.

MMTTs in older animals. After weaning at 5-6 weeks, both CF and control animals were maintained on a slurry of canned food (Halo) and Elecare (Abbott Laboratories) supplemented with pancreatic enzymes. MMTTs were performed in weaned animals 45-90 days of age using the following protocol optimized for reproducibility in juvenile ferrets. After being allowed to feed until satiated, animals were fasted for 3 hours prior to the MMTT. Blood glucose was measured during the fast at $-90,-60,-30$, and 0 minutes before the MMTT. The MMTT was initiated by feeding the animals a quantity of Elecare and canned food normalized to a body surface area estimate (BSAE, in $\mathrm{cm}^{2}$ ) of each animal: $(\mathrm{BW}[\mathrm{g}] \times \text { body length }[\mathrm{cm}])^{0.5}$. Each MMTT was performed in pairs with aged-matched CF and non-CF animals using the same ratio of food to BSAE (typically $0.2 \mathrm{ml}$ Elecare per $\mathrm{cm}^{2}$ BSAE and $0.27 \mathrm{~g}$ solid food per $\mathrm{cm}^{2}$ BSAE was used). Postprandial blood samples were then collected at 15, 30, 60, 90, and 120 minutes. Blood glucose concentrations were determined immediately using a portable glucose meter (OneTouch LifeScan).
Measurements of plasma insulin, C-peptide, and cortisol. Plasma insulin was measured using an ELISA kit that is specific for bovine and porcine fully processed insulin and does not cross-react with proinsulin or C-peptide (Calbiotech). Plasma C-peptide was measured using a human/rat/mouse C-peptide-specific Enzyme Immunoassay Kit (RayBio) based on the principle of competitive enzyme immunoassay. Plasma cortisol was measured using an ELISA kit (Calbiotech). To evaluate the total insulin content of the pancreas, newborn kits were weighed and immediately sacrificed after birth, and the pancreas was dissected. Whole pancreas wet weight was recorded, and then pancreas protein extracts were prepared by acetic acid extraction as previously described (50). Extracts were used for insulin ELISA measurements and total protein measurements. Protein concentrations were determined using the Bio-Rad Protein Assay.

Measurements of acute and late-phase insulin secretion. Acute insulin secretion tests were performed on 6- to 12-hour-old kits that had been fasted for 3 hours using L-arginine and glucose stimulation tests as previously described, with minor modifications (71). Kits were injected (i.p.) with glucose $(3 \mathrm{~g} / \mathrm{kg} \mathrm{BW})$ or L-arginine $(0.3 \mathrm{~g} / \mathrm{kg} \mathrm{BW})$. Blood glucose levels were measured from a pin prick to the tail immediately before and 2 minutes after the injection. Plasma samples were then immediately collected into chilled heparinized tubes for insulin ELISA measurements. Plasma samples were immediately frozen and stored at $-80^{\circ} \mathrm{C}$ prior to analysis. For analysis of late-phase insulin secretion, 3-hour-fasted 6-to 12-hour-old kits were injected (i.p.) with glucose $(2 \mathrm{~g} / \mathrm{kg} \mathrm{BW})$, and blood was harvested at 30,60 , and 120 minutes after injection for blood glucose and plasma insulin determinations as described above.

Neonatal islet culture and insulin secretion studies. CF and non-CF islets were isolated and cultured using a previously described protocol for neonatal pig islets (50). Briefly, pancreata from 3-5 CF or non-CF kits were minced into 1 - to $2-\mathrm{mm}^{3}$ pieces and incubated in HBSS containing $0.5 \% \mathrm{BSA}, 1 \%$ penicillin/streptomycin, and $2.5 \mathrm{mg} / \mathrm{ml}$ collagenase for 4 minutes at $37^{\circ} \mathrm{C}$. Following digestion, the tissue fragments were washed 3 times in medium without collagenase and then cultured in 24-well plates with $2 \mathrm{ml} \mathrm{Ham}$ 's F12 medium (containing $10 \mathrm{mM}$ glucose) supplemented with $0.5 \% \mathrm{BSA}$, $50 \mu \mathrm{M}$ 3-isobutyl-1-methylxanthine (IBMX), $10 \mathrm{mM}$ nicotinamide, $2 \mathrm{mM}$ L-glutamine, and $100 \mathrm{U} / \mathrm{ml}$ penicillin and $100 \mu \mathrm{g} / \mathrm{ml}$ streptomycin. The medium was changed on the second day after isolation and every other day thereafter. On the ninth day of culture, approximately 500-600 islets were selectively removed with a small glass pipette, washed, and divided into 2 equal-sized matched groups for each genotype and then incubated in RPMI 1640 containing $2 \mathrm{mM}$ glucose, $2 \mathrm{mM}$ L-glutamine, and 0.5\% BSA for 1 hour at $37^{\circ} \mathrm{C}$. To initiate the insulin secretion experiment, islets were then transferred into $1 \mathrm{ml}$ of fresh RPMI 1640 medium containing $2 \mathrm{mM}$ or $30 \mathrm{mM}$ glucose for 1 hour at $37^{\circ} \mathrm{C}$. The media and islets were then collected, and total insulin was quantified in both by ELISA. The amount of insulin secreted into the media during the 1-hour period and that remaining in the islets was used to calculate the percent insulin secretion $(\%$ insulin secretion = secreted insulin in the media/total insulin in the media and islets at the end of the experiment). SI was calculated as percent insulin secretion at $30 \mathrm{mM}$ glucose divided by percent insulin secretion at $2 \mathrm{mM}$ glucose.

Insulin-mediated AKT signaling. Newborn 6- to 12-hour-old ferrets were fasted for 3 hours and then anesthetized with isofluorane. The hepatic portal vein was surgically exposed and $1.5 \mathrm{U} / 10 \mathrm{~g} \mathrm{BW}$ insulin was injected in a volume of $60-100 \mu \mathrm{l}$ over approximately 10 seconds. Animals were then euthanized at 5 minutes after insulin injection, and skeletal muscle, liver, and perirenal fat were rapidly excised. The tissue samples were immediately snap-frozen in liquid nitrogen and stored at $-80^{\circ} \mathrm{C}$ until use. Tissues were processed in lysis reagent (C2978, Sigma-Aldrich) containing 1\% (v/v) protease (P8340, Sigma-Aldrich) and phosphatase (524625, Calbiochem) inhibitor cocktails. Lysates (70 $\mu \mathrm{g}$ protein) were blotted using antibodies 
against AKT and phospho-AKT Ser473 (9272, 9271, Cell Signaling Technology). Filters were first probed with the phospho-AKT Ser473 antibody, then stripped and reprobed with the total AKT antibody. The ratio of phospho-AKT to total AKT immunoreactivity was assessed for each animal using NIH Image. To avoid subtle differences in exposure times between experiments, tissues from $8 \mathrm{CF}$ and 8 non-CF animals were equally divided for two Western blot experiments ( $4 \mathrm{CF}$ and 4 non-CF animals) that probed for phospho-AKT and total AKT in each organ at the same time. An internal insulin-treated reference control from each tissue in the first experiment was loaded onto gels of the second experiment and used to normalize subtle differences in exposure times between the two experiments.

Bile plug quantification in the liver. Liver sections from newborn 6- to 12-hour-old non-CF and CF ferrets were scored for bile plugs within hepatic canaliculi and bile ducts. Scoring for bile canalicular plugs was performed on two liver sections per animal by counting the total number of canalicular plugs (scored as any visible accumulation of bile) in five $\times 400$ fields per section. The average number of canalicular plugs per tissue section was used to determine the canalicular bile plug score for each animal. The percentage of bile duct plugs was determined by counting the bile ducts containing bile plugs (any visible accumulation of bile) and dividing by the total number of bile ducts per section. Two sections were counted in their entirety, and the percentages were averaged for each animal. To minimize counting multiple profiles of the same bile duct, each portal area was counted as one bile duct.

Hepatic triglyceride analysis. Total triglyceride content of the liver was measured using a previously described protocol (72). Newborn 6- to 12-hourold ferrets were fasted for 3 hours and euthanized, and the liver was rapidly excised and immediately snap-frozen in liquid nitrogen and stored at $-80^{\circ} \mathrm{C}$ until use. Briefly, a defined mass of liver tissue $(\sim 200 \mathrm{mg})$ was homogenized in $350 \mu \mathrm{l}$ ethanolic potassium hydroxide, followed by incubation at $55^{\circ} \mathrm{C}$ overnight until no oil layer was visible. The sample was brought up to $1 \mathrm{ml}$ with $50 \%$ ethanol, votexed, and then centrifuged at $4^{\circ} \mathrm{C}$ for 5 minutes. The supernatant was removed to a new tube, topped off to $1.2 \mathrm{ml}$ with $50 \%$ ethanol, and then vortexed. Two hundred microliters of that sample was then mixed with $215 \mu \mathrm{l}$ of $1 \mathrm{M} \mathrm{MgCl}_{2}$, vortexed, and incubated on ice for 10 minutes prior to centrifugation at $4^{\circ} \mathrm{C}$ for 5 minutes. The supernatant was then removed to a new tube and assayed for triglycerides using Free Glycerol Reagent (F6428, Sigma-Aldrich) and Glycerol Standards (G7793, Sigma-Aldrich) to construct standard curves. The total triglyceride $(\mathrm{mg})$ per gram liver was then calculated.

Statistics. Data are expressed as mean \pm SEM of $n$ independent observations or animals. Individual GTT time points and GTT AUC, insulin, C-peptide, and cortisol comparisons between CF and non-CF animals were evaluated using the nonparametric 2-tailed Mann-Whitney $U$ test. A 2-tailed $F$ test was used to compare variances in values of CF and non$\mathrm{CF}$ datasets. Statistical analysis of regression slopes and intercepts for $\log$ [insulin] versus glucose plots used the linear model function in $\mathrm{R}$ (http://www.R-project.org). For studies involving older animals with CF and non-CF paired groups, multiple variance components were modeled in a mixed-effects model evaluating longitudinal analysis and repeated- measures analysis for changes in blood glucose with age and MMTT blood glucose values after feeding. These analyses were conducted using the lme 4 package for the R statistical computing environment. In the analysis of the age-dependent trends in $\mathrm{CF} /$ non-CF blood glucose ratios of older animals (3 paired groups), the log of the ratio of blood glucose of a CF animal to that of its matched non-CF animal was computed, and a mixed-effects model was then fitted, with age the fixed effect and animal pair the random effect. For MMTT data on paired CF/non-CF groups, we conducted two analyses on the log of blood glucose using a mixed-effects model. In both analyses, the effect of animals was treated as random, and the group effect of each experiment was treated as fixed. In the first analysis, time after feeding was included as a fixed effect with a random slope. This analysis assessed whether post-feeding blood glucose was significantly different between CF and non-CF paired groups using aggregate data from the MMTT experiments. In the second analysis, the focus was on the effect of CF versus non-CF status at every time point during the MMTT assay using aggregate data from the MMTT experiments for the paired groups. Morphometric analysis assessing 3 or more datasets was evaluated using Kruskal-Wallis nonparametric 1-way ANOVA with a Dunn's posttest. Morphometric analysis assessing 2 datasets was evaluated using the nonparametric 2-tailed Mann-Whitney $U$ test. Percent insulin secretion from islets of a single genotype was evaluated using the nonparametric 2-tailed paired Wilcoxon test, while differences in the SI of insulin between genotypes was evaluated using the nonparametric 2-tailed Mann-Whitney $U$ test. Differences in percent insulin secretion of islets between genotypes at low and high glucose were evaluated using Kruskal-Wallis nonparametric 1-way ANOVA with a Dunn's post-test. In all statistical analyses, $P$ values less than 0.05 were deemed significant.

Study approval. This study was performed according to protocols approved by the Institutional Animal Care and Use Committee of the University of Iowa.

\section{Acknowledgments}

This work was supported by NIH grants DK047967, DK091211, HL108902, HL099516 (to J.F. Engelhardt), DK092284 (to Z.A. Stewart), DK081548 and Fraternal Order of Eagles Diabetes Research Center grant (to A.W. Norris), and the University of Iowa Center for Gene Therapy (DK54759). We also gratefully acknowledge Scott Blackman (Johns Hopkins University) for helpful discussions during the course of this work.

Received for publication September 1, 2011, and accepted in revised form July 26, 2012.

Address correspondence to: John F. Engelhardt, Room 1-111 BSB, Department of Anatomy and Cell Biology, College of Medicine, University of Iowa, 51 Newton Road, Iowa City, Iowa 52242, USA. Phone: 319.335.7744; Fax: 319.335.6581; E-mail: john-engelhardt@ uiowa.edu. Or to: Andrew W. Norris, 1270 CBRB, 285 Newton Road, University of Iowa, Iowa City, Iowa 52242, USA. Phone: 319. 356.4443; Fax: 319.356.8170; E-mail: andrew-norris@uiowa.edu.
1. Moran A, et al. Epidemiology, pathophysiology, and prognostic implications of cystic fibrosisrelated diabetes: a technical review. Diabetes Care. 2010;33(12):2677-2683.

2. Hameed S, Jaffe A, Verge CF. Cystic fibrosis related diabetes (CFRD) - the end stage of progressive insulin deficiency. Pediatr Pulmonol. 2011;46(8):747-760.

3. Lanng S, Thorsteinsson B, Nerup J, Koch C. Influence of the development of diabetes mellitus on clinical status in patients with cystic fibrosis. Eur J Pediatr. 1992;151(9):684-687.

4. Bismuth E, et al. Glucose tolerance and insulin secretion, morbidity, and death in patients with cystic fibrosis. J Pediatr. 2008;152(4):540-545.e1.

5 . Koch C, et al. Presence of cystic fibrosis-related diabetes mellitus is tightly linked to poor lung function in patients with cystic fibrosis: data from the European Epidemiologic Registry of Cystic Fibrosis. Pediatr Pulmonol. 2001;32(5):343-350.

6. Brodsky J, Dougherty S, Makani R, Rubenstein RC, Kelly A. Elevation of 1-hour plasma glucose during oral glucose tolerance testing is associated with worse pulmonary function in cystic fibrosis. Diabetes Care. 2011;34(2):292-295.
7. Cheung MS, et al. Growth in children with cystic fibrosis-related diabetes. Pediatr Pulmonol. 2009; 44(12):1223-1225.

8. Moran A, Dunitz J, Nathan B, Saeed A, Holme B, Thomas W. Cystic fibrosis-related diabetes: current trends in prevalence, incidence, and mortality. Diabetes Care. 2009;32(9):1626-1631.

9. Ripa P, Robertson I, Cowley D, Harris M, Masters IB, Cotterill AM. The relationship between insulin secretion, the insulin-like growth factor axis and growth in children with cystic fibrosis. Clin Endocrinol (Oxf). 2002;56(3):383-389. 
10. Moran A, Doherty L, Wang X, Thomas W. Abnormal glucose metabolism in cystic fibrosis. J Pediatr. 1998;133(1):10-17.

11. De Schepper J, Hachimi-Idrissi S, Smitz J, Dab I, Loeb H. First-phase insulin release in adult cystic fibrosis patients: correlation with clinical and biological parameters. Horm Res. 1992;38(5-6):260-263.

12. Mohan K, et al. Mechanisms of glucose intolerance in cystic fibrosis. Diabet Med. 2009;26(6):582-588.

13. Mohan K, Israel KL, Miller H, Grainger R, Ledson MJ, Walshaw MJ. Long-term effect of insulin treatment in cystic fibrosis-related diabetes. Respiration. 2008;76(2):181-186.

14. Dobson L, Hattersley AT, Tiley S, Elworthy S, Oades PJ, Sheldon CD. Clinical improvement in cystic fibrosis with early insulin treatment. Arch Dis Child. 2002;87(5):430-431.

15. Moran A, et al. Insulin sensitivity in cystic fibrosis. Diabetes. 1994;43(8):1020-1026.

16. Durie PR, Forstner GG. Pathophysiology of the exocrine pancreas in cystic fibrosis. J R Soc Med. 1989;82(suppl 16):2-10.

17. Iannucci A, Mukai K, Johnson D, Burke B. Endocrine pancreas in cystic fibrosis: an immunohistochemical study. Hum Pathol. 1984;15(3):278-284.

18. Lohr M, et al. Cystic fibrosis associated islet changes may provide a basis for diabetes. An immunocytochemical and morphometrical study. Virchows Arch A Pathol Anat Histopathol. 1989;414(2):179-185.

19. O'Riordan SM, Dattani MT, Hindmarsh PC. Cystic fibrosis-related diabetes in childhood. Horm Res Paediatr. 2010;73(1):15-24.

20. Moran A, Phillips J, Milla C. Insulin and glucose excursion following premeal insulin lispro or repaglinide in cystic fibrosis-related diabetes. Diabetes Care. 2001;24(10):1706-1710.

21. Street ME, et al. Insulin production and resistance in cystic fibrosis: effect of age, disease activity, and genotype. J Endocrinol Invest. 2012;35(3):246-253.

22. Moran A, et al. Clinical care guidelines for cystic fibrosis-related diabetes: a position statement of the American Diabetes Association and a clinical practice guideline of the Cystic Fibrosis Foundation, endorsed by the Pediatric Endocrine Society. Diabetes Care. 2010;33(12):2697-2708.

23. Austin A, Kalhan SC, Orenstein D, Nixon P, Arslanian S. Roles of insulin resistance and beta-cell dysfunction in the pathogenesis of glucose intolerance in cystic fibrosis. J Clin Endocrinol Metab. 1994;79(1):80-85.

24. Hardin DS, LeBlanc A, Para L, Seilheimer DK. Hepatic insulin resistance and defects in substrate utilization in cystic fibrosis. Diabetes. 1999; 48(5):1082-1087.

25. Wilmshurst EG, et al. Endogenous and exogenous insulin responses in patients with cystic fibrosis. Pediatrics. 1975;55(1):75-82.

26. Lombardo F, et al. Natural history of glucose tolerance, beta-cell function and peripheral insulin sensitivity in cystic fibrosis patients with fasting euglycemia. Eur J Endocrinol. 2003;149(1):53-59.

27. Lanng S, Thorsteinsson B, Roder ME, Nerup J, Koch C. Insulin sensitivity and insulin clearance in cystic fibrosis patients with normal and diabetic glucose tolerance. Clin Endocrinol (Oxf). 1994;41(2):217-223.

28. Yung B, Noormohamed FH, Kemp M, Hooper J, Lant AF, Hodson ME. Cystic fibrosis-related diabetes: the role of peripheral insulin resistance and betacell dysfunction. Diabet Med. 2002;19(3):221-226.

29. Cucinotta $D$, et al. No changes of insulin sensitivity in cystic fibrosis patients with different degrees of glucose tolerance: an epidemiological and longitudinal study. Eur J Endocrinol. 1994;130(3):253-258.

30. van Haren EH, Hopman WP, Jansen JB, Rosenbusch
G, Lamers CB, van Herwaarden CL. Increased plasma cholecystokinin levels and small gall bladders in adult patients with cystic fibrosis. Clin Sci (Lond). 1991;81(1):85-89.

31. Kuo P, et al. Gastric emptying, incretin hormone secretion, and postprandial glycemia in cystic fibrosis - effects of pancreatic enzyme supplementation. J Clin Endocrinol Metab. 2011;96(5):E851-E855.

32. Ross SA, Morrison D, McArthur RG. Hypersecretion of gastric inhibitory polypeptide in nondiabetic children with cystic fibrosis. Pediatrics. 1981; 67(2):252-254.

33. Poretsky L. Principles of Diabetes Mellitus. 2nd ed. Boston, Massachusetts, USA: Springer; 2010.

34. Sauerwein HP, Schols AM. Glucose metabolism in chronic lung disease. Clin Nutr. 2002;21(5):367-371.

35. Stalvey MS, Flotte TR. Endocrine parameters of cystic fibrosis: back to basics. J Cell Biochem. 2009; 108(2):353-361.

36. Stalvey MS, et al. Cystic fibrosis transmembrane conductance regulator deficiency exacerbates islet cell dysfunction after beta-cell injury. Diabetes. 2006; 55(7):1939-1945.

37. Fisher JT, Zhang Y, Engelhardt JF. Comparative biology of cystic fibrosis animal models. Methods Mol Biol. 2011;742:311-334.

38. Keiser NW, Engelhardt JF. New animal models of cystic fibrosis: what are they teaching us? Curr Opin Pulm Med. 2011;17(6):478-483.

39. Sun X, et al. Adeno-associated virus-targeted disruption of the CFTR gene in cloned ferrets. J Clin Invest. 2008;118(4):1578-1583.

40. Sun X, et al. Disease phenotype of a ferret CFTRknockout model of cystic fibrosis. J Clin Invest. 2010; 120(9):3149-3160.

41. Lindblad A, Glaumann H, Strandvik B. Natural history of liver disease in cystic fibrosis. Hepatology. 1999;30(5):1151-1158.

42. Scher H, Bishop WP, McCray PB. Ursodeoxycholic acid improves cholestasis in infants with cystic fibrosis. Ann Pharmacother. 1997;31(9):1003-1005.

43. Lepage $\mathrm{G}$, et al. Ursodeoxycholic acid improves the hepatic metabolism of essential fatty acids and retinol in children with cystic fibrosis. J Pediatr. 1997;130(1):52-58.

44. Solomon MP, et al. Glucose intolerance in children with cystic fibrosis. J Pediatr. 2003;142(2):128-132.

45. Larsson H, Ahren B. Glucose-dependent arginine stimulation test for characterization of islet function: studies on reproducibility and priming effect of arginine. Diabetologia. 1998;41(7):772-777.

46. Pan FC, Wright C. Pancreas organogenesis: from bud to plexus to gland. Dev Dyn. 2011;240(3):530-565.

47. Garibaldi BA, Pecquet Goad ME, Fox JG, Sylvina TJ, Murray R. Serum cortisol radioimmunoassay values in the normal ferret and response to ACTH stimulation and dexamethasone suppression tests. Lab Anim Sci. 1988;38(4):452-454.

48. Duckworth WC, Bennett RG, Hamel FG. Insulin degradation: progress and potential. Endocr Rev. 1998;19(5):608-624.

49. Michael MD, et al. Loss of insulin signaling in hepatocytes leads to severe insulin resistance and progressive hepatic dysfunction. Mol Cell. 2000; 6(1):87-97.

50. Korbutt GS, Elliott JF, Ao Z, Smith DK, Warnock GL, Rajotte RV. Large scale isolation, growth, and function of porcine neonatal islet cells. J Clin Invest. 1996;97(9):2119-2129.

51. Morino K, et al. Reduced mitochondrial density and increased IRS- 1 serine phosphorylation in muscle of insulin-resistant offspring of type 2 diabetic parents. J Clin Invest. 2005;115(12):3587-3593.

52 . Lee J, et al. Multiple abnormalities of myocar- dial insulin signaling in a porcine model of dietinduced obesity. Am J Physiol Heart Circ Physiol. 2010;298(2):H310-H319.

53. Gutierrez-Juarez R, et al. Critical role of stearoylCoA desaturase-1 (SCD1) in the onset of dietinduced hepatic insulin resistance. J Clin Invest. 2006; 116(6):1686-1695.

54. Morino K, et al. Muscle-specific IRS-1 Ser->Ala transgenic mice are protected from fat-induced insulin resistance in skeletal muscle. Diabetes. 2008; 57(10):2644-2651.

55. Minicucci L, et al. Liver disease as risk factor for cystic fibrosis-related diabetes development. Acta Paediatr. 2007;96(5):736-739.

56. Colombo C. Liver disease in cystic fibrosis. Curr Opin Pulm Med. 2007;13(6):529-536.

57. Lykavieris P, Bernard O, Hadchouel M. Neonatal cholestasis as the presenting feature in cystic fibrosis. Arch Dis Child. 1996;75(1):67-70.

58. Feranchak AP, Sokol RJ. Cholangiocyte biology and cystic fibrosis liver disease. Semin Liver Dis. 2001;21(4):471-488.

59. Smith BW, Adams LA. Nonalcoholic fatty liver disease and diabetes mellitus: pathogenesis and treatment. Nat Rev Endocrinol. 2011;7(8):456-465.

60. Del Prato S, Tiengo A. The importance of firstphase insulin secretion: implications for the therapy of type 2 diabetes mellitus. Diabetes Metab Res Rev. 2001;17(3):164-174.

61. Elder DA, Wooldridge JL, Dolan LM, D'Alessio DA. Glucose tolerance, insulin secretion, and insulin sensitivity in children and adolescents with cystic fibrosis and no prior history of diabetes. J Pediatr. 2007;151(6):653-658.

62. Anzeneder L, Kircher F, Feghelm N, Fischer R, Seissler J. Kinetics of insulin secretion and glucose intolerance in adult patients with cystic fibrosis. Horm Metab Res. 2011;43(5):355-360.

63. Battezzati A, et al. Spontaneous hypoglycemia in patients with cystic fibrosis. Eur J Endocrinol. 2007; 156(3):369-376.

64. Radike K, Molz K, Holl RW, Poeter B, Hebestreit H, Ballmann M. Prognostic relevance of hypoglycemia following an oral glucose challenge for cystic fibrosis-related diabetes. Diabetes Care. 2011;34(4):e43.

65 . Sturgess JM. Structural and developmental abnormalities of the exocrine pancreas in cystic fibrosis. J Pediatr Gastroenterol Nutr. 1984;3(suppl 1):S55-S66.

66. Imrie JR, Fagan DG, Sturgess JM. Quantitative evaluation of the development of the exocrine pancreas in cystic fibrosis and control infants. Am J Pathol. 1979;95(3):697-708.

67. Raman VS, et al. Hyperglycemia and diabetes mellitus in children with pancreatitis. J Pediatr. 2011; 158(4):612-616e1.

68. Lucidi $V$, et al. The etiology of acute recurrent pancreatitis in children: a challenge for pediatricians. Pancreas. 2011;40(4):517-521.

69. Walkowiak J, Lisowska A, Blaszczynski M. The changing face of the exocrine pancreas in cystic fibrosis: pancreatic sufficiency, pancreatitis and genotype. EurJ Gastroenterol Hepatol. 2008;20(3):157-160.

70. Segal I, et al. Cystic fibrosis transmembrane conductance regulator ion channel function testing in recurrent acute pancreatitis. J Clin Gastroenterol. 2008;42(7):810-814.

71. Kulkarni RN, et al. beta-cell-specific deletion of the Igf1 receptor leads to hyperinsulinemia and glucose intolerance but does not alter beta-cell mass. Nat Genet. 2002;31(1):111-115.

72. Norris AW, et al. Muscle-specific PPARgamma-deficient mice develop increased adiposity and insulin resistance but respond to thiazolidinediones. J Clin Invest. 2003;112(4):608-618. 